\title{
me
}

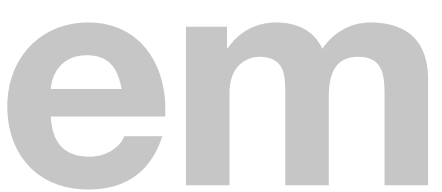

\section{magazine of european medical oncology}

Official Journal of the Central European Cooperative Oncology Group (CECOG) and Austrian Society for Haematology and Medical Oncology (OeGHO)

memo (2021) 14 (Suppl 2):S23-S41

https://doi.org/10.1007/s12254-021-00697-2

Online publiziert: 16 March 2021

(C) Springer-Verlag GmbH Austria, part of Springer

Nature 2021

OeGHO

Österreichische Gesellschaft für

Hämatologie \& Medizinische Onkologie

\section{Proceedings of the Annual Meeting of the Austrian Society of Haematology and Medical Oncology}

\section{Frühjahrstagung 2021}

der Österreichischen Gesellschaft für Hämatologie und Medizinische Onkologie und der AHOP - Arbeitsgemeinschaft hämatologischer und onkologischer Pflegepersonen in Österreich

Wien, 8.-10. April 2021

\section{Tagungspräsident:}

Prim. Univ.-Prof. Dr. Wolfgang Hilbe

\section{Tagungssekretariat:}

OA Priv.-Doz. Dr. Daniel Heintel

OÄ Priv.-Doz. Dr. Kathrin Strasser-Weippl, MBA

1. Medizinische Abteilung, Zentrum für Onkologie und Hämatologie mit Ambulanz und Palliativstation Klinik Ottakring, Wien 



\section{Poster Hämatologie}

\section{H01}

\section{CAR mediates differentiation and migration in erythroid precursor cells and is specifically down-regulated in MDS}

\section{Karin Bauer ${ }^{\star 1,2}$, Sigrid Machherndl-}

Spandl' ${ }^{3}$ Lukas Kazianka', Sinan Gültekin', Susanne Suessner ${ }^{4}$, Johannes Proell ${ }^{4}$, Jeroen Lauf ${ }^{5}$, Gregor Hoermann ${ }^{2,6}$, Gregor Eisenwort ${ }^{1,2}$, Norman Häfner ${ }^{7}$, Mathilde FödermayrMayrleitner ${ }^{3}$, Ann-Sofie Schmolke ${ }^{1}$, Emiel van der Kouwe ${ }^{1}$, Uwe Platzbecker,9, ${ }^{8,9}$ Thomas Lion ${ }^{10}$, Ansgar Weltermann ${ }^{3}$, Otto Zach $^{3}$, Gerald Webersinke ${ }^{3}$, Ulrich Germing ${ }^{11}$, Marie C. Béné ${ }^{12}$, Wolfgang R. Sperr ${ }^{1,2}$, Christian Gabriel ${ }^{4}$, Philipp B. Staber ${ }^{1,2}$, Peter Bettelheim ${ }^{3,5}$, Peter Valent ${ }^{1,2}$

${ }^{1}$ Medical University of Vienna, Vienna, Austria ${ }^{2}$ Ludwig Boltzmann Institute for Hematology and Oncology, Vienna, Austria

${ }^{3}$ Ordensklinikum Barmherzige Schwestern Linz, Linz, Austria ${ }^{4}$ Transfusion Service of Upper Austria, Linz, Austria ${ }^{5}$ Labor Europaplatz, Linz, Austria

${ }^{6}$ MLL Munich Leukemia Laboratory, Munich, Germany

${ }^{7}$ Jena University Hospital, Jena, Germany

¿University of Dresden, Dresden, Germany

'University Hospital Leipzig, Leipzig, Germany

${ }^{10}$ Children's Cancer Research Institute Vienna, Vienna,

Austria

${ }^{11}$ Heinrich-Heine-University, Düsseldorf, Germany

${ }^{12} \mathrm{CHU}$ de Nantes, Nantes, France

Myelodysplastic syndromes (MDS) are myeloid neoplasms characterized by an accumulation of dysplastic erythroid cells in the bone marrow (BM) and peripheral anemia. The etiology underlying the block in migration and maturation of erythroid progenitor cells (EryPC) in MDS remains unknown. We screened for genes that are expressed abnormally in EryPC. In mRNA array- and flow cytometry validation experiments, we found that the Coxsackie-Adenovirus Receptor (CAR) is downregulated in CD45low/CD105+ EryPC in patients with MDS. Correspondingly, the erythroblast cell lines HEL, K562 and KU812 stained negative for CAR. Lentiviral transduction of fulllength CAR into these cells resulted in an increased expression of erythroid differentiation antigens, including CD36, CD71, and glycophorin $\mathrm{A}$ as well as in an increased migration against a serum protein-gradient, whereas truncated CAR-variants did not induce erythroid antigens or migration. Finally, conditional knock-out of Car in hematopoietic cells in C57BL/6 mice resulted in anemia and erythroid dysplasia. Together, CAR is a functionally relevant marker that is down-regulated on EryPC in MDS and is of prognostic significance. In summary, our data show that decreased CAR expression contributes to the maturation defect and altered migration of EryPC and thus to the pathogenesis of MDS.
Expression of $A B C C 1$ modulates the response of acute myeloid leukemia (AML) cells to BCL2 inhibition

Jessica Ebner ${ }^{\star 1}$, Martin Piontek ${ }^{1}$, Gabriele Manhart ${ }^{1}$, Gergely Szakacs ${ }^{3}$, Johannes Schmöllerl², Florian Grebien ${ }^{1}$

${ }^{1}$ Institute for Medical Biochemistry, University of Veterinary Medicine, Vienna, Austria

2IMP - Research Institute of Molecular Pathology, Vienna, Austria

${ }^{3}$ Institute for Cancer Research, Medical University, Vienna, Austria

Background: The BCL2 inhibitor Venetoclax is a promising targeted anti-cancer agent for the treatment of AML. Despite its overall success, the understanding of molecular mechanisms that modulate Venetoclax efficacy is of utmost importance for successful personalized therapy. ATP-binding cassette (ABC-)transporters were strongly implicated in cancer chemotherapy resistance, including AML, but their function in normal AML physiology as well as their context in Venetoclax therapy was unknown. We hypothesize that ABC-transporters act as critical modulators of Venetoclax efficiency.

Methods: We used a combination of CRISPR/Cas9-enabled functional genomics, pharmacological and cell biological approaches in human AML cell lines to systematically evaluate genetic requirements for $\mathrm{ABC}$-transporters in drug-naïve $\mathrm{AML}$, as well as analyze how particular ABC-transporters control Venetoclax sensitivity of AML cells.

Results: Using CRISPR/Cas9-mediated knockout of all 48 ABC-transporters in MOLM13 cells, we revealed that only 4 were essential for cell growth. However, we found that knockout of ABCC1 strongly increased the sensitivity of AML cells to Venetoclax. Genetic and pharmacological ABCC1 inhibition sensitized AML cells to the BCL2-inhibitors Navitoclax, ABT-737 and AZD4320, but not to other drugs that are used to treat AML. ABCC1 overexpression induced Venetoclax resistance in leukemia cells. MCL1-overexpressing Venetoclax-resistant AML cells could be re-sensitized to BCL2 inhibition by ABCC1 inhibition.

Conclusions: Currently, we are investigating the molecular mechanisms underlying the synergy between $\mathrm{ABCC} 1$ and Venetoclax. In addition to novel molecular insights, our findings could help to establish ABCC1 expression as a biomarker that helps to predict the efficiency of Venetoclax in the treatment of AML. 


\section{H03 Gewinner Jubiläumspreis}

\section{Targeting constitutively active TYK2 in acute lymphoblastic leukemia}

\section{Katharina Wöss ${ }^{\star 1}$, Caroline Lassnig ${ }^{1,2}$, Andrea Pölzl|',2, Katrin Meiss| ${ }^{1}$, Tania Brandstötter ${ }^{3}$, Veronika Sexl ${ }^{3}$, Roland Kuiper ${ }^{4}$, Miriam Butler ${ }^{4}$, Dorette van Ingen Schenau ${ }^{4}$, Frank van Leeuwen ${ }^{4}$, Thomas Rülicke ${ }^{2,5}$, Richard Moriggl', Florian Grebien ${ }^{6}$, Birgit Strobl', Sabine Macho-Maschler ${ }^{1,7}$, Mathias Müller ${ }^{1,2}$}

${ }^{1}$ Institute of Animal Breeding and Genetics, University of Veterinary Medicine Vienna, Vienna, Austria

${ }^{2}$ University Center Biomodels Austria, University of Veterinary Medicine Vienna, Vienna, Austria

Institute of Pharmacology and Toxicology, University of Veterinary Medicine Vienna, Vienna, Austria

${ }^{4}$ Princess Máxima Center for Pediatric Oncology, Utrecht, The Netherlands

Insitute of Laboratory Animal Science, University of Veterinary Medicine Vienna, Vienna, Austria ${ }^{6}$ Institute of Medical Biochemistry, University of Veterinary Medicine Vienna, Vienna, Austria

${ }^{7}$ Unit of Physiology, Pathophysiology and Experimental Endocrinology, Vienna, Austria

Background: Sequencing unravels molecular mechanisms of leukemogenesis and identifies treatment targets. Non-receptor tyrosine kinase 2 (TYK2) belongs to the Janus kinase (JAK) family and governs responses to cytokines and growth factors. Two germline mutations of TYK2 (P760L, G761V) were found in patients developing several de novo leukemias and potentially predispose to leukemia.

Methods: We studied oncogenic transformation, signalling pathway interaction and efficacy of therapeutic approaches using cell lines, primary cells and patient derived xenograft (PDX) cells expressing mutated TYK2. Additionally, we generated a mouse model enabling cell type specific expression of tagged human TYK2P760L and TYK2WT which will allow in vivo studies of molecular functions and interaction partners.

Results: In all cell systems, both mutations led to activated TYK2 and downstream activation of signal transducers and activators of transcription (STAT) 1, 3 and 5. TYK2P760L facilitated colony formation and cytokine independent growth of diverse hematopoietic cell lines and primary cells. It also caused leukemia development in xenograft mouse models. We employed a screen of 700 kinase inhibitors and identified the PI3K/AKT/ mTOR pathway to cooperate with TYK2P760L. TYK2 and PI3K/ AKT/mTOR inhibitors acted synergistically in cell lines and PDX cells. Preliminary results indicate that germline expression of TYK2WT and TYK2P760L allows the birth of normally developed offspring with TYK2P760L conferring constitutive TYK2 phosphorylation and a skin hyperproliferative phenotype.

Conclusions: We demonstrate a role of TYK2 in leukemogenesis and offer a new treatment approach for cancer with an aberrant activation of TYK2.

The work is funded by the Austrian Science Fund FWF SFB-F61 and DK-W1212.

\section{HO4}

Phenotypic characterization of human basophils and mast cells reveals expression of various virus receptors, immune checkpoint antigens and Siglec-molecules

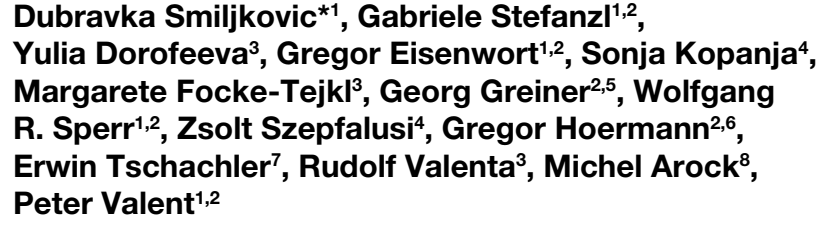

${ }^{1}$ Division of Hematology and Hemostaseology, Department of Internal Medicine I, Medical University of Vienna, Vienna, Austria

${ }^{2}$ Ludwig Boltzmann Institute for Hematology and Oncology, Medical University of Vienna, Vienna, Austria

${ }^{3}$ Division of Immunopathology, Department of Pathophysiology and Allergy Research, Center for Pathophysiology, Immunology and Infectiology, Medical University of Vienna, Vienna, Austria

${ }^{4}$ Department of Pediatrics and Adolescent Medicine, Medical University of Vienna, Vienna, Austria

${ }^{5} \mathrm{hr}$ Labor, Medical Diagnostic Laboratories, Vienna, Austria ${ }^{6}$ Munich Leukemia Laboratory (MLL), Munich, Germany ${ }^{7}$ Department of Dermatology, Medical University of Vienna, Vienna, Austria

${ }^{8}$ Department of Hematology, Hôpital Pitié-Salpêtrière and Sorbonne University, Paris, France

Basophils (BA) and mast cells (MC) are effector cells of allergic reactions. Both cells express numerous surface-receptors that may play a role in migration, adhesion and/or activation of BA and MC. In this study, we provide an update of our CD-profiling studies of human BA and MC. Primary blood BA were obtained from healthy donors, allergic individuals and patients with chronic myeloid leukemia (CML). Primary MC were isolated from lung, skin, normal/reactive bone marrow (BM) and patients with systemic mastocytosis (SM). The BA cell line KU812 and the MC lines HMC-1, ROSA, and MCPV were also examined. Expression of $\mathrm{CD}$ antigens was analyzed by multi-color flow cytometry. Primary BA and MC were found to display several virus-receptors, including the corona virusreceptor AP-N (CD13), measles virus-receptor MCP (CD46), rhinovirus-receptor ICAM-1 (CD54), echovirus-receptor DAF (CD55), PVRL2=Nectin-2 (CD112), and HIV-co-receptor CXCR4 (CD184). Primary BA and MC also expressed various activation-linked or inhibitory surface antigens, such as MRGPRX2, LMIR1 (CD300a) or LAIR1 (CD305). Moreover, primary $\mathrm{BA}$ and $\mathrm{MC}$ expressed various immune checkpoint antigens (CD47, CD81, CD274=PD-L1) and Siglec-molecules, including Siglec-1, Siglec-3, Siglec-7, and Siglec-10. The BA and MC lines tested exhibited a similar profile of $C D$ antigens when compared to primary cells. We also found that CML BA express higher levels of CD13, CD81, CD312, CD317, Siglec-6, Siglec-8, and Siglec-10 compared to normal BA. Antibody-based phenotyping provides further evidence that $\mathrm{BA}$ and $\mathrm{MC}$ are separate hematopoietic lineages. Both types of cells display a number of virus receptors, activation-linked antigens, immune checkpoint antigens and Siglec-molecules. 
$\mathrm{N}$-terminal fragment of brain natriuretic peptide (pBNP) is an individual prognostic marker for survival in patient with AML undergoing intensive chemotherapy

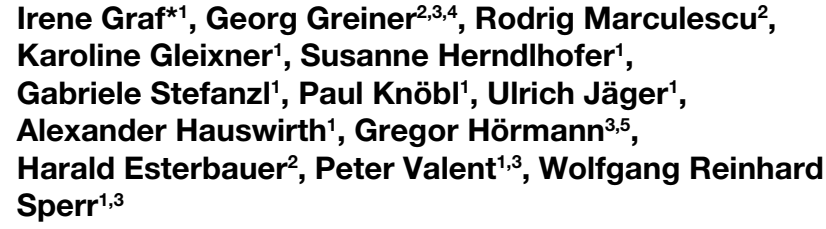

1Division of Hematology and Hemostaseology, Department of Internal Medicine I, Medical University of Vienna, Vienna, Austria

${ }^{2}$ Department of Laboratory Medicine, Medical University of Vienna, Vienna, Austria

${ }^{3}$ Ludwig Boltzmann Institute for Hematology and Oncology, Medical University of Vienna, Vienna, Austria

${ }^{4} \mathrm{hr}$ Labor, Medical Diagnostic Laboratories, Vienna, Austria ${ }^{5}$ MLL Munich Leukemia Laboratory, Munich, Germany

Background: Karyotype, genomic abnormalities and patient-related factors are known to play an important role in the prognostication of AML. Little is known about the effects of serologic markers like $\mathrm{N}$-terminal fragment of brain natriuretic peptide (pBNP) on the outcome of AML patients.

Methods: We analyzed AML patients treated since 11/1994 with $3+7$ based induction and consolidation using up to 4 cycles of intermediate-dose or high-dose ARA-C.

Results: pBNP levels at diagnosis were available in 252 patients. In 163 patients (63.7\%) elevate pBNP levels were detectable and in 99 patients (36.3\%), pBNP levels were within normal range $(0-125 \mathrm{pg} / \mathrm{ml})$. In 14 patients $(5.5 \%)$, pBNP exceeded $2000 \mathrm{pg} / \mathrm{mL}$. There was a rough correlation between age and $\mathrm{pBNP}(\mathrm{R}=0.19)$ and no significant difference between male and female patients $(p=0.7)$. Following induction therapy 175 patients $(71 \%)$ achieved a complete remission, $52(6.1 \%)$ had no remission (NR), and 17 (7\%) had early death (ED). pBNP levels differed significantly among CR, NR and ED patients, with 153,207 , and $715 \mathrm{pg} / \mathrm{mL}$, respectively $(p=0.001)$. Overall survival (OS) and continuous complete remission (CCR) also differed significantly between patients with normal, moderately elevated $(125-2000 \mathrm{pg} / \mathrm{mL})$ and highly elevated pBNP $(>2000 \mathrm{pg} / \mathrm{mL})$ (OS: $p<0.001$; CCR: $p<0.029)$. This was also observed when analyzing OS in patients $\leq 60$ years and $>60$ years separately. In multivariate analysis assessing age, karyotype, FLT3 ITD and NPM-1, pBNP remained an independent predictive variable for OS $(p=0.023)$ but not for CCR $(p=0.16)$.

Conclusions: Together, $\mathrm{pBNP}$ is an independent prognostic factor and biomarker indicating the risk of early death and survival in AML.
Repetitive relapse and repetitive remission after multiple chemotherapy-courses in AML: Are there patients who may benefit and can hope for prolonged survival?

\section{Irene Graf*1, Georg Greiner ${ }^{2,3,4}$, Karoline Gleixner ${ }^{1}$, Susanne Herndlhofer ${ }^{1}$, Gabriele Stefanzl ${ }^{1}$, Paul Knöbl', Alexander Hauswirth', Ulrich Jäger', Gregor Hörmann ${ }^{3,5}$, Peter Valent ${ }^{1,3}$, Wolfgang Reinhard Sperr ${ }^{1,3}$}

${ }^{1}$ Division of Hematology and Hemostaseology, Department of Internal Medicine I, Medical University of Vienna, Vienna, Austria

${ }^{2}$ Department of Laboratory Medicine, Medical University of Vienna, Vienna, Austria

${ }^{3}$ Ludwig Boltzmann Institute for Hematology and Oncology, Medical University of Vienna, Vienna, Austria ${ }^{4} \mathrm{hr}$ Labor, Medical Diagnostic Laboratories, Vienna, Austria ${ }^{5}$ MLL Munich Leukemia Laboratory, Munich, Germany

Background: Without transplantation, survival of patients with relapsed AML after chemotherapy is poor. However, some patients may benefit from re-induction chemotherapy and enter a second or even third complete remission (CR).

Methods and Results: We identified 24 patients who received multiple courses of intensive poly-chemotherapy (MCR-AML) with subsequent consolidation using up to 4 cycles of intermediate-dose or high-dose ARA-C. All patients entered $\mathrm{CR}$ for at least 3 times and/or had relapse-free intervals of at least 1 year. The median age was 64 (38-76) years. At diagnosis, median white blood count (WBC) was $4.65 \mathrm{G} / \mathrm{L}$. No MCR-AML patient had a WBC $>50 \mathrm{G} / \mathrm{L}$. Fifteen patients $(62.8 \%)$ presented with intermediate, $6(25 \%)$ with unfavorable and $2(8 \%)$ with a favorable karyotype by SWOG criteria. Median continuous CR was $2.3(0.8-7.1)$ years, and median durations of second and third CR were $1.2(0.5-6.5)$ and $0.8(0.4-1.2)$ years, respectively. Of all patients, $71 \%$ achieved 4 CR and $12.5 \%$ achieved 5 CR. Median overall survival was $6.3(1.8-14.3)$ years. Comorbidities were detected in most patients. According to the HCT-CI, 5 patients $(21 \%)$ were in the low, $10(42 \%)$ in the intermediate, and $9(37 \%)$ in the high-risk group.

Conclusions: Together, a small group of AML patients may benefit from multiple courses of intensive chemotherapy despite multiple relapses. Longer time-intervals between relapses, and CR after one re-induction-cycle may increase the likelihood that the patient will benefit from this therapy. Whether additional post-CR maintenance with hypomethylating agents can further prolong survival in these patients remains to be determined in forthcoming studies. 


\section{H07 Gewinner Jubiläumspreis}

\section{Identification of essential direct transcriptional target genes of NUP98-JARID1A in acute myeloid leukemia}

\section{Selina Tröster*1, Johannes Schmoellerl ${ }^{2}$, Thomas Eder ${ }^{1}$, Georg Winter ${ }^{3}$, Johannes Zuber ${ }^{2}$, Florian Grebien}

${ }^{1}$ Institute for Medical Biochemistry, University of Veterinary Medicine, Vienna, Austria

${ }^{2}$ Research Institute of Molecular Pathology - IMP, Vienna, Austria

${ }^{3}$ CeMM Research Center for Molecular Medicine of the Austrian Academy of Sciences, Vienna, Austria

Background: The Nucleoporin 98 (NUP98) gene is recurrently involved in the formation of oncogenic fusion proteins in acute myeloid leukemia (AML). Expression of NUP98-fusions is associated with poor prognosis, particularly in pediatric patients. As no targeted therapy exists, there is an urgent need for a better understanding of the molecular mechanisms of NUP98-fusion-protein-dependent leukemogenesis to empower the development of tailored treatments.

Methods: To investigate functional genetic dependencies we conducted a genome-scale CRISPR/Cas9 loss-of-function screen in a cellular model of NUP98-JARID1A-dependent AML. Next we reasoned that a detailed understanding of the underlying transcriptional circuitries is critical to understand NUP98fusion-dependent gene regulation. Using a newly developed model for ligand-induced degradation of the NUP98-JARID1A protein via the degradation tag (dTAG) system and performing nascent RNA-seq (SLAM-seq), we uncovered immediate transcriptional changes upon acute NUP98-JARID1A degradation.

Results: With the dTAG system, complete NUP98-JARID1A protein degradation was achieved within one hour after ligand addition, leading to terminal differentiation and apoptosis of leukemia cells. SLAM-seq analysis revealed that 45 genes were significantly down-regulated upon NUP98-JARID1A degradation. By intersecting these candidates with 2550 genes that were critical for NUP98-JARID1A-AML cell proliferation and survival in the CRISPR/Cas9 screen, 12 high-confidence essential direct targets of NUP98-JARID1A were identified.

Conclusions: This approach allows the identification of functional genetic dependencies and direct transcriptional target genes of NUP98-JARID1A in AML. Multi-layered validations of high-confidence candidates in a variety of models, including primary patient samples, will provide new insights into the molecular mechanism of NUP98-JARID1A-driven leukemia and might identify novel actionable gene targets.
Effects of avapritinib and nintedanib on growth and survival of KIT D816V+ human mast cells

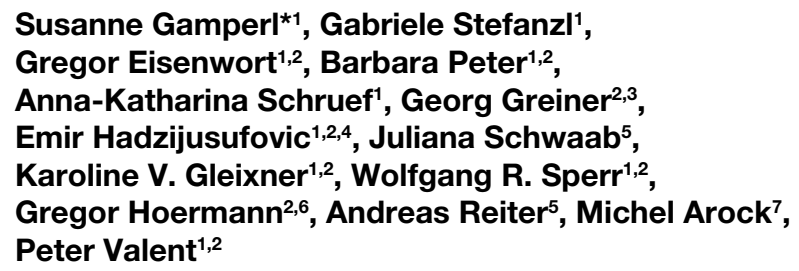

${ }^{1}$ Division of Hematology and Hemostaseology, Department of Internal Medicine I, Medical University of Vienna, Vienna, Austria

${ }^{2}$ Ludwig Boltzmann Institute of Hematology and Oncology, Medical University of Vienna, Vienna, Austria

${ }^{3} \mathrm{hr}$ Labor, Medical Diagnostic Laboratories, Vienna, Austria ${ }^{4}$ Department/Hospital for Companion Animals and Horses, University Hospital for Small Animals, Internal Medicine Small Animals, University of Veterinary Medicine Vienna, Vienna, Austria

${ }^{5}$ Department of Hematology and Oncology, University Medical Centre Mannheim and Medical Faculty Mannheim, Heidelberg University, Germany

${ }^{6}$ Munich Leukemia Laboratory (MLL), Munich, Germany ${ }^{7}$ Department of Hematological Biology, Pitié-Salpêtrière Hospital, Pierre et Marie Curie University (UPMC), Paris, France

Systemic mastocytosis (SM) is a rare disease characterized by abnormal growth and accumulation of neoplastic mast cells (MC) in various organ-systems. In the majority of SM patients, the D816V-mutated variant of the stem cell factor receptor, KIT, which mediates ligand-independent activation and autonomous growth of MC, can be detected. Several tyrosine kinase inhibitors targeting KIT D816V have recently been developed, but many patients with advanced SM develop resistance. We evaluated the effects of two novel KIT D816V-targeting drugs, avapritinib and nintedanib, on growth and survival of primary neoplastic MC and various human MC lines. Avapritinib was found to suppress growth of HMC-1.1 (KIT V560G+) and HMC-1.2 cells (KIT V560G+ and D816V+) with comparable $\mathrm{IC}_{50}$ values $(0.1-0.25 \mu \mathrm{M})$. In addition, avapritinib was found to block proliferation of $\operatorname{ROS}^{\mathrm{A}} \mathrm{KIT}^{\mathrm{WT}}\left(\mathrm{IC}_{50}\right.$ : $\left.0.1-0.25 \mu \mathrm{M}\right)$, ROS $^{\mathrm{AKIT}}$

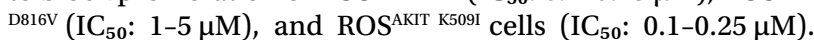
Stronger effects were seen with nintedanib IC $_{50}$ in HMC-1.1:

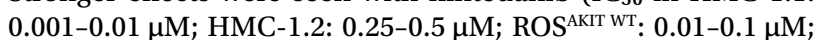

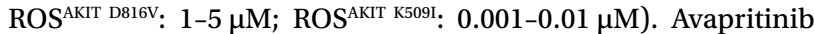
and nintedanib also suppressed the growth of primary neoplastic MC in most patients with advanced SM (IC I $_{50}$ obtained with avapritinib: $1-5 \mu \mathrm{M} ; \mathrm{IC}_{50}$ produced by nintedanib: 0.1-5 $\left.\mu \mathrm{M}\right)$. Growth inhibition was accompanied by apoptosis induction and downregulation of expression of CD63, CD71 and/or CD117 (KIT) in neoplastic MC. Both drugs failed to suppress IgE-dependent histamine release in MC or basophils. Together, our data suggest that avapritinib and nintedanib are promising drugs for treatment of advanced SM. 
Characterization of marker and target expression profiles in CD34+/CD38- and CD34+/CD38+ stemand progenitor cells in $\mathrm{AML}$ and $\mathrm{CML}$

\author{
Irina Sadovnik ${ }^{\star 1,2}$, Harald Herrmann ${ }^{1,2,3}$, \\ Gregor Eisenwort', Thomas Rülicke, ${ }^{1,4}$, \\ Katharina Blatt ${ }^{1,2}$, Susanne Herndlhofer ${ }^{1,2}$, \\ Michael Willmann ${ }^{1,5}$, Gabriele Stefanzl ${ }^{2}$, \\ Georg Greiner ${ }^{1,6}$, Axel Schulenburg ${ }^{1,7}$, \\ Niklas Mueller ${ }^{2}$, Werner Rabitsch ${ }^{7}$, Martin Bilban ${ }^{1,8}$, \\ Gregor Hoermann ${ }^{1,9}$, Wolfgang Sperr ${ }^{1,2}$, Peter Valent ${ }^{1,2}$
}

${ }^{1}$ Ludwig Boltzmann Institute for Hematology and Oncology, Medical University of Vienna, Vienna, Austria

2Division of Hematology and Hemostaseology, Department of Internal Medicine I, Medical University of Vienna, Vienna, Austria

${ }^{3}$ Department of Radiation Oncology, Medical University of Vienna, Vienna, Austria

${ }^{4}$ Institute of Laboratory Animal Science, University of Veterinary Medicine Vienna, Vienna, Austria ${ }^{5}$ Department of Companion Animals and Horses, Clinic for Internal Medicine and Infectious Diseases, University of Veterinary Medicine Vienna, Vienna, Austria

${ }^{6} \mathrm{hr}$ Labor, Medical Diagnostic Laboratories, Vienna, Austria ${ }^{7}$ Department of Internal Medicine I, Stem Cell Transplantation Unit, Medical University of Vienna, Vienna, Austria

${ }^{8}$ Department of Laboratory Medicine, Medical University of Vienna,Vienna, Austria

${ }^{9}$ MLL Munich Leukemia Laboratory, Munich, Germany

In order to identify novel markers and immunological targets in leukemic stem cells (LSC) in acute myeloid leukemia (AML) and chronic myeloid leukemia (CML), we analyzed bone marrow samples of patients with AML $(n=274)$, CML $(n=97)$, and controls $(n=288)$ for the expression of surface antigens on CD34+/ CD38- and CD34+/CD38+ cells by multi-color flow cytometry. In addition, we established mRNA expression profiles in highly purified, sorted CD34+/CD38- and CD34+/CD38+ cells by gene array- and qPCR studies. We were able to identify aberrantly expressed markers in all patient cohorts examined. CD34+/ CD38- LSC in CML exhibited an almost invariable aberrationprofile defined as CD25+/CD26+/CD56+/CD93+/IL-1RAP+. AML LSC variably expressed 'aberrant' surface antigens, including CD25 (48\%), CD96 (40\%), CD371 (CLL-1) (68\%), and IL-1RAP ( $65 \%)$. With the exception of a subgroup of FLT3 ITD-mutated patients, AML LSC did not exhibit CD26. All other surface markers and target-antigens detected on AML and/or CML LSC, including CD33, CD44, CD47, CD52, CD105, CD114, CD117, CD133, CD135, CD184 and roundabout-4 (ROBO4), were also found to be expressed on normal bone marrow stem cells. However, various of these surface targets, including CD25, CD33 and CD123 were expressed at higher levels on CD34+/ CD38- LSC compared to normal bone marrow stem cells. Furthermore, antibody-mediated immunological targeting through CD33 and/or CD52 resulted in LSC depletion in vitro and in a substantially reduced LSC engraftment in NSG mice. Together, we have established surface marker and target expression-profiles of AML and CML LSC, which should facilitate LSC enrichment, diagnostic LSC phenotyping and development of LSC-eradicating immunotherapies.
Brusatol and F3AK treatment of aggressive lymphoma cells results in induction of caspase independent apoptosis

Sonja Jantscher ${ }^{\star 1}$, Sandra Haingartner ${ }^{1}$, Katrin Pansy ${ }^{1}$, Hildegard Greinix ${ }^{1}$, Peter Neumeister ${ }^{1}$, Thomas Erker ${ }^{2}$, Burkhard Klösch ${ }^{3}$, Alexander Deutsch ${ }^{1}$

'Division of Haematology, Department of Internal Medicine, Medical University of Graz, Graz, Austria

2Department of Pharmaceutical Chemistry, University of Vienna, Vienna, Austria

${ }^{3}$ Ludwig Boltzmann Institute for Rheumatology and Balneology, Graz, Austria

Background: DLBCL is the most common lymphoma entity in adults. The fact that the incidence of DLBCL is still increasing and that approximately one-third of patients relapses or does not attain remission, indicates the requirement to develop novel therapeutic strategies. Therefore, we aimed to determine the effect of Brusatol and F3AK known to possess anti-tumoral properties for both on aggressive lymphoma cells.

Methods: Therefore, we cultured seven different lymphoma cell lines (Karpas422 and SuDHL4 as DLBCL-GCB models, RI-1 and U2932 as DLBCL-ABC models, BL-2 and Raji as Burkitt lymphoma model, and Jurkat as model for T-NHLs) with Brusatol or F3AK followed by MTS assays to determine cell growth, cell cycle analysis and apoptosis assays (Annexin V-staining, caspase 3 cleavage, and PARP-cleavage).

Results: After 72 hours of cell exposure to Brusatol or F3AK in culture, concentration-dependent growth inhibition in all investigated cell lines was observed. In six B cell lymphoma cell lines, Brusatol treatment was associated with lower IC50 values (range $22 \mathrm{nM}-685 \mathrm{nM}$ ) compared to F3AK treatment (range $675 \mathrm{nM}-2073 \mathrm{nM}, p=0.005$ ). Furthermore, culturing of cells with both agents resulted in more than $50 \%$ of lymphoma cells staining positive for Annexin $\mathrm{V}$ and exhibited a SubGl one peak after $24 \mathrm{~h}$ for Brusatol in a concentration of 1000, 250, and 50 $\mathrm{nM}$ and F3AK $1000 \mathrm{nM}$. Interestingly, no caspase 3 cleavage was observed in none of the two treatments.

Conclusions: These preclinical data indicate that Brusatol or F3AK induce caspase-independent apoptosis. Thus, both agents should be considered when developing novel anti-lymphoma therapies.

\section{H11}

\section{Diphenyleneiodonium possesses cell growth} inhibitory effects on aggressive lymphoma cells

Sandra Haingartner ${ }^{\star 1}$, Sandra Jantscher ${ }^{1}$, Katrin Pansy ${ }^{1}$, Peter Neumeister ${ }^{1}$, Hildegard Geinix ${ }^{1}$, Burkhard Klösch ${ }^{2}$, Alexander Deutsch ${ }^{1}$

'Division of Haematology, Department of Internal Medicine, Medical University of Graz, Graz, Austria

${ }^{2}$ Ludwig Boltzmann Institute for Rheumatology and Balneology, Graz, Austria

Background: Diffuse large B-cell lymphoma (DLBCL) are lymphomas with pronounced genetic and clinical heterogeneity. Transcription profiling identified a DLBCL subgroup that displayed increased expression of genes involved in mitochon- 
drial oxidative phosphorylation (OXPHOS). Since this subgroup is insensitive to B-cell-receptor (BCR) inhibition, we aim to investigate whether inhibition of oxidative phosphorylation could be used as a therapeutic target.

Methods: Therefore, we used seven different lymphoma cell lines (Karpas422 and SuDHL4 as DLBCL-GCB models, RI-1 and U2932 as DLBCL-ABC models, BL-2 as Burkitt lymphoma model and Jurkat as model for T-cell non-Hodgkin lymphoma (NHL). These cells were cultured with Diphenyleneiodonium (DPI)-an OXPHOS inhibitor-followed by cell growth as well as cell cycle analysis and apoptosis assays (Annexin V and cleaved caspase 3 staining).

Results: After 72 hours of DPI exposure in cell culture, concentration-dependent growth inhibition in all investigated cell lines was detected by MTS assay. Especially, DLBCL cell lines exhibited low IC50 values ( $31 \mathrm{nM}, 22 \mathrm{nM}, 40 \mathrm{nM}$, and $12 \mathrm{nM}$ for RI-1, U2932, Karpas422 and SuDHL4), whereas IC50 values for $\mathrm{BL}-2(149 \mathrm{nM})$ and Jurkat $(640 \mathrm{nM})$ cells were higher $(p=0.071)$. Furthermore, DPI treatment $(250 \mathrm{nM}, 50 \mathrm{nM}$ and $10 \mathrm{nM})$ of SuDHL4, U2932 and BL2 cells induced a G0/G1 cell cycle arrest, whereas no apoptotic effects were detected.

Conclusions: These preclinical data indicate that DPI treatment causes cell growth inhibition, which is mediated by G0/G1 cell cycle arrest in aggressive lymphoma cells, especially DLBCL cells. Thus, pharmacologic inhibition of OXPHOS might serve as a novel therapeutic approach for further lymphoma therapies.

\section{H12}

First safety assessment after first dosage application of the BNT162b2 mRNA Covid-19 Vaccine in hematologic and oncologic patients

\section{Beatrix Mutschlechner ${ }^{* 1,3}$, Magdalena Benda ${ }^{* 1,3}$, Michele Atzl ${ }^{1}$, Luciano Severgnini', Andreas Volgger ${ }^{1}$, Patrick Reimann', Klaus Gasser', Bernd Hartmann', Monika Lenzi', Minh Huynh', Thomas Winder ${ }^{1,2}$}

${ }^{1}$ Academic Teaching Hospital Feldkirch, Feldkirch, Austria ${ }^{2}$ University of Zurich, Zurich, Switzerland

${ }^{3}$ Private University in the Principality of Liechtenstein, Triesen, Principality of Liechtenstein

Background: Several studies have shown that cancer patients are more likely to suffer from severe events in case of a SARS-CoV-2 infection. Currently, vaccination is the best evaluated preventive strategy. To our knowledge, there are no studies to date that examined the safety and efficacy of the BNT162b2 mRNA Covid-19 Vaccine in cancer patients.

Methods: We retrospectively analysed 86 hemato-oncologic patients who received the first part of BNT162b2 mRNA Covid19 vaccine in January 2021. We assessed side effects within 7 days after vaccination by a standardized questionnaire in accordance with the registration study by Polack et al.

Results: In our cohort, $67.4 \%$ of patients were male and the mean age was 68 years. $53.5 \%$ of patients had hematologic diseases, $45.3 \%$ of patients had solid tumors, $1.2 \%$ suffering from both. $95 \%$ of solid tumor patients had metastatic cancer. Patients received either systemic treatment $(74.5 \%)$ or were under close surveillance for advanced cancer or hematologic disease $(25.6 \%)$. $39.5 \%$ of patients reported adverse events, of which $88.1 \%$ were grade $1,9.5 \%$ were grade 2 and $2.4 \%$ were grade 3 . No grade 4 event occurred. The most commonly reported reactions were muscle pain at injection site and fatigue ( $16.3 \%$ and $11.6 \%$, respectively). No significant difference was found regarding to gender, disease entity or therapy ( $p>0.05$, respectively).

Conclusions: Early safety results after first BNT162b2 mRNA Covid-19 vaccination are in accordance with the registration study by Polack et al. No new safety issues were observed in this early safety analysis. Final safety analysis will be presented at the meeting.

\section{H13 Gewinner Jubiläumspreis}

Impact of the skin microbiome in graft-versushost disease development after allogeneic hematopoietic stem cell transplantation

Nadine Bayer*1, Bela Hausmann², Petra Pjevac', Laura-Marie Gail', Ram VinayPandey ${ }^{1}$, Johanna Strobl', Marion $\mathrm{Nehr}^{3}$, Martin Watzenböck', Margo Jentus' ${ }^{1}$, Lukas Hammerl', Athanasios Makristathis ${ }^{3}$, David Berry ${ }^{2}$, Georg Stary ${ }^{1}$

${ }^{1}$ Department of Dermatology, Medical University of Vienna, Vienna, Austria

2DOME (Division of Microbial Ecology), University of Vienna, Vienna, Austria

${ }^{3}$ Department of Laboratory Medicine, Microbiology, Medical University of Vienna, Vienna, Austria

${ }^{4} \mathrm{CeMM}$ (Research Center for Molecular Medicine), Vienna, Austria

${ }^{5}$ Ludwig Boltzmann Institute for Rare and Undiagnosed Diseases, Vienna, Austria

Background: The success of allogeneic hematopoietic stem cell transplantation (HSCT) remains limited due to severe side effects, such as graft-versus-host disease (GVHD). Dysbiosis of intestinal microbes has already been linked to GVHD development. However, the role of the skin microbiome remains elusive.

Methods: Patient material (stool, skin scrapes) collected at 5 time points before and up to one year after HSCT was evaluated for risk-associated species correlating with GVHD development $(n=44)$ by $16 \mathrm{~S}$ sequencing. Additionally, bulk-RNA seq was performed from cutaneous T cells (CD3+CD20-HLA-DR-) and dendritic cells/macrophages (CD3-CD20-HLA-DR+) of the same patient cohort. Finally, host microbe interactions were assessed via immunofluorescence stainings as well as co-culture experiments.

Results: The intestinal microbiome of HSCT patients shows a clear disruption following the day of transplantation (d0), which is restored at later time points. In comparison, the cutaneous microbiome remains more stable over time and upon HSCT. Yet, 16S rRNA FISH in skin biopsies of the same patients revealed a sharp decrease in bacteria/mm2 on day 0 and 14 after HSCT. In the gut, an increase in the relative abundance of Enterococcus spp. already at day of transplantation served as a predictor for GVHD development. Skin GVHD lesions presented with an increased colonization of Staphylococcus spp. and showed a dose-dependent induction of inflammatory cytokines when co-cultivated with keratinocytes. Further, T cells showed upregulation of NF-kappaB signaling and cellular defense against bacteria in the early phase post-HSCT (14 days).

Conclusions: To conclude, the microbiome is drastically disturbed in HSCT and may contribute to GVHD. 
H14

COVID-19 bei hämatoonkologisch Vorerkrankten eine retrospektive Analyse

Michael Leiner*1, Michael Panny*1, Jan Miechowiecki', Ulla Seyfried $^{2}$, Ruth Kariotis ${ }^{2}$, Barbara Lehner ${ }^{1}$, Sonja Klenkhart ${ }^{1}$, Jochen Zwerina ${ }^{2}$, Johann Sipötz ${ }^{3}$, Felix Keil ${ }^{1}$

13. Medizinische Abteilung, Hanusch-Krankenhaus, Wien, Österreich

21. Medizinische Abteilung, Hanusch-Krankenhaus, Wien, Österreich

32. Medizinisch Abteilung, Wien, Österreich

Grundlagen: Im Hanusch-Krankenhaus wurde im Oktober 2020 angesichts der COVID-19-Pandemie eine Covid-Station mit Schwerpunktversorgung für hämato-/onkologische Patientinnen und Patienten eröffnet. In einer Metaanalyse mit 3377 COVID-19-Erkrankten (Vijenthira et al., Blood 2020) ist eine Mortalität von $34 \%$ bei COVID-19-Fällen mit hämatologischer Vorerkrankung beschrieben.

Methodik: Retrospektive Analyse der hämatologischen Fälle mit COVID-19. Die Daten wurden zum Vergleich von vorliegenden epidemiologischen Zahlen mit denen einer hämatologisch spezialisierten Covid-Station erhoben.

Ergebnisse: Von 12.10 .2020 bis 01.02 .2021 wurden insgesamt 101 Männer und 91 Frauen mit PCR-avider SARS-CoV2-Infektion aufgenommen. Das mediane Alter lag bei 71 Jahren. Unter 192 Aufnahmen gab es 32 Todesfälle (16,7\%), bei $56 \mathrm{kam}$ es zu einem milden, bei $81 \mathrm{zu}$ einem moderaten und bei $45 \mathrm{zu}$ einem schweren Verlauf. Eine maligne Diagnose bestand bei 73 Aufnahmen ( 54 unter Therapie). Von 35 hämatologisch Erkrankten unter laufender Therapie entwickelten 13 einen unkomplizierten (37,1 \%), $18(51,4 \%)$ einen moderaten, 4 $(11,4 \%)$ einen schweren Verlauf.

Die Odds Ratio für einen schweren Verlauf lag für maligne Erkrankungen bei 0,79, im Vergleich dazu für pulmonale Begleiterkrankungen bei 2,72 und für Vorliegen einer Kardiomyopathie bei 2,35. Die durchschnittliche Liegedauer lag bei 10,6 gegenüber 8,7 Tagen mit bzw. ohne maligne Erkrankung, bei 11,1 gegenüber 8,9 Tagen mit bzw. ohne hämatologische Erkrankung.

Schlussfolgerungen: Wie vorbeschrieben, zeigen sich protrahierte COVID-19-Verläufe innerhalb eines hämatologisch komorbiden Patientenkollektivs. Outcomes anhand preliminärer Daten erscheinen bei responsiver hämatologischer Grunderkrankung nicht aggraviert im Vergleich zu anderen COVID-19-Verläufen. Aktualisierte Daten werden am Kongress präsentiert.

\section{H15}

Unravelling survival strategies of proteasome inhibitor (PI) resistant multiple myeloma (MM) cells

\section{Anja Schneller ${ }^{* 1}$, Niklas Zojer ${ }^{1}$, Arnold Bolomsky ${ }^{1}$, Heinz Ludwig ${ }^{1}$}

${ }^{1}$ Wilhelminen Krebsforschung GmbH - Klinik Ottakring, Vienna, Austria

Background: Despite new therapy strategies (i. e. PI; IMiDs) most MM patients suffer from refractory disease and run out of therapy options. This limitation is chiefly linked to the occurrence of drug resistance during the course of the disease. The apoptosis pathway is regulated by the $\mathrm{BH} 3$ protein family. A delicate interplay within a complex network of protein-proteininteractions is key to navigate cell survival and cell death. Studies showed that this is influenced by PI resistance, indicating that the dysregulation of the BH3-proteins is one of the key survival mechanisms in PI resistant cells. Here, we aim to reveal and characterize central mechanisms of apoptosis evasion in response to the PIs Carfilzomib and Ixazomib.

Methods: We generated human myeloma cell lines with acquired carfilzomib $(n=9)$ and ixazomib $(n=4)$ resistance, which were examined by the unbiased flow cytometry-based profiling method iBH3 to reveale the dependency on one or more specific BH3-proteins as well as protein expression analysis.

Results: The generation of PI resistant cell lines led to a $>10$-fold shift in IC50 values. Protein expression analysis of BCL2 family members in carfilzomib sensitive and resistant cell lines highlighted cell line specific alterations of distinct pro- and anti-apoptotic proteins. Moreover, iBH3 revealed the dependencies on anti-apoptotic BH3 proteins and indicated a switch of BH3 dependencies in PI resistant vs sensitive cells.

Conclusions: These results underline a potential survival strategy of PI resistant MM cells and will prompt us to an indepth analysis of the apoptosis signaling pathway in PI resistant cells.

\section{H16}

The impact of ATG-based in-vivo T-cell depletion on cytomegalovirus reactivation after stem cell transplantation depends on the number of HLA-C group 1 (C1) KIR ligands

\section{Alexander Nikoloudis ${ }^{\star 1}$, Johannes Clausen ${ }^{1,2}$, Sigrid Machherndl-Spandl1,2, Veronika Buxhofer- Ausch $^{1,2}$, Irene Strassl' ${ }^{1}$, Olga Stiefel', Dagmar Wipplinger'1, Robert Milanov', Emine Kaynak', Petra Hasengruber ${ }^{1}$, Ansgar Weltermann ${ }^{1,2}$, Andreas Petzer ${ }^{1}$, Dominik Wolf ${ }^{3}$, David Nachbaur ${ }^{3}$}

${ }^{1}$ Department of Internal Medicine I: Hematology with Stem Cell Transplantation, Hemostaseology and Medical Oncology, Ordensklinikum Linz - Elisabethinen, Linz, Austria ${ }^{2}$ Medical Faculty, Johannes Kepler University, Linz, Austria ${ }^{3}$ University Hospital of Internal Medicine V, Hematology \& Oncology, Medical University of Innsbruck, Innsbruck, Austria

Background: Anti-thymocyte globulin (ATG) is an established agent for prevention of severe graft-versus-host disease (GVHD) after hematopoietic stem cell transplantation (HSCT). The beneficial effect of ATG was shown to depend on the number of HLA-C KIR-ligand group 1 (C1) alleles, significantly decreasing non-relapse mortality (NRM), without significantly affecting the relapse incidence, in $\mathrm{Cl}+$ recipients (Clausen et al., Biomedicines 2017). We postulated that the impact of ATG on cytomegalovirus (CMV) reactivation may similarly depend on the number of $\mathrm{Cl}$ alleles.

Methods: Retrospectively, we analyzed 530 HLA-matched related or unrelated HSCT for hematological malignancies, using bone marrow or peripheral blood, and at-risk for CMV (recipient and/or donor CMV seropositive). ATG-Fresenius was applied in 211 transplants. Multivariate analyses were 
performed by competing risk regression. Univariate analyses applied the cumulative incidence function and the gray's test. CMV reactivation was defined as PCR or pp65Ag positivity resulting in preemptive therapy.

Results: CMV reactivation occurred in 279 HSCT recipients. In recipients with $\mathrm{C} 1 / \mathrm{C} 1$ KIR-L status, ATG strongly increased the incidence of CMV reactivation (sHR 3.2; $\mathrm{P}<0.0001 ; n=194$ ). A less pronounced effect of ATG was seen in $\mathrm{C} 1 / \mathrm{C} 2$ recipients (sHR1.6; $\mathrm{P}=0.005 ; n=253$ ). By contrast, in recipients with $\mathrm{C} 2 / \mathrm{C} 2$ KIR-L status, ATG was not associated with an increase of CMV reactivations (sHR0.74; $\mathrm{P}=0.49 ; n=83$ ).

Conclusions: These findings clearly show a significant interaction between ATG treatment and the recipient's HLA-C KIR-L status in post-transplant CMV surveillance, and may help further elucidate the role of CMV in graft-versus leukemia effects after HSCT.

\section{H17}

Rituximab and prednisone with or without radiotherapy in elderly frail patients with diffuse large B-cell lymphoma - a case series

\section{Karl Mayrhofer ${ }^{\star 1}$, Wolfgang Hibe ${ }^{1}$, Daniel Heintel ${ }^{1}$}

${ }^{1}$ Klinik Ottakring, Vienna, Austria

Background: Treating elderly and frail patients with DLBCL is a unique therapeutic challenge since standard chemotherapy is not applicable. We report the outcome of patients deemed unable to tolerate chemotherapy who were treated with 3-weekly rituximab $\left(375 \mathrm{mg} / \mathrm{m}^{2}\right)$ plus prednisone $(100 \mathrm{mg} /$ day for five days) with or without radiotherapy at our institute between 2012 and 2020.

Patient characteristics: Nine patients were identified. Median age was 83 years (range 75-93) and ECOG performance status ranged between 2 and 4 . Severe comorbidities were present in five patients.

Results: Median number of administered rituximab cycles was 6 (range 1-9) and radiotherapy was applied to five patients. While four of our patients had no clinical benefit, five profited notably from therapy. Partial remission was achieved in four patients and one patient who had localized primary cutaneous DLBCL responded with a complete remission. Median OS was 10 months (range 1 day -3 years) and median PFS was 3.2 months. Median PFS in responders and non-responders was 25.1 months and 1 month, respectively. Positive outcomes were associated with ECOG performance status $<4$, non-severe comorbidities, more localized disease, intermediate risk by IPI (as opposed to high risk) and those who received radiation to the main tumor mass.

Conclusions: Although the small number of patients in this case series limits its expressiveness, it seems that selected frail and elderly patients with DLBCL who are unable to tolerate any chemotherapy potentially profit from therapy with rituximab plus prednisone.

\section{H18}

Effects of the nuclear orphan receptor Nr4a1 on
immune evasion in aggressive lymphomas

\section{Doreen Truntschnig*1, Katrin Pansy², Alexander Deutsch ${ }^{2}$}

${ }^{1}$ Fachhochschule Kärnten, Klagenfurt am Wörthersee, Austria ${ }^{2}$ Division of Hematology, Department of Internal Medical University of Graz, Graz, Austria

Background: For the development of new therapeutic strategies, a research group at the Medical University of Graz were conducting studies on the nuclear receptor Nr4al in aggressive lymphomas. The data of their studies identified Nr4al as a putative tumor suppressor. Preclinical models have shown that loss of Nr4al accelerates lymphoma development in vivo and leads to activation of inhibitory immune receptors of the B7/ CD28 families. These data support the hypothesis that Nr4al is significantly involved in the regulation of immune evasion of aggressive lymphomas via the B7/CD28 axis. The aim of this study was to comprehensively investigate the impact of Nr4al on receptors and genes being implicated in immune activation, inhibition and associated with T-cell exhaustion.

Methods: The methodological approach of this work is based on an RNA gene expression analysis of 63 immunoregulatory genes by means of semi-quantitative real-time PCR of aggressive lymphomas. The expression levels of these immunregulatory genes were compared between lymphomas with and without Nr4al loss using transgenic mouse model.

Results: The results showed that seven genes were significantly differentially expressed in the Nr4a1-/- tumors. Higher expression was noted for the inhibitory receptor CD244, the immunoregulatory genes Jumonji and LDHA-Alpha and the immunofunction associated genes Cbl-b, NFATC1 and TOX. SOCS2 was significantly downregulated.

Conclusions: Nr4al has an impact on genes involved in immune regulation and genes which are related to the state of T-cell exhaustion in tumors. However, further investigations are necessary to make more precise statements.

\section{H19}

Tackling pyrimidine biosynthesis in multiple myeloma (MM) - Evaluation of the novel dihydroorotate dehydrogenase (DHODH) inhibitor PTC299 as anti-myeloma treatment strategy

\section{Christina Pfeiffer ${ }^{\star 1}$, Arnold Bolomsky ${ }^{1}$, Niklas Zojer ${ }^{1}$, Martin Schreder ${ }^{1}$, Heinz Ludwig ${ }^{1}$}

${ }^{1}$ Department of Medicine I, Wilhelminen Cancer Research Institute, Klinik Ottakring, Vienna, Austria

Background: Clinical progress in patients with high-risk $\mathrm{MM}$ is limited and median survival remains at less than two years for this patient subgroup. While the demand for pyrimidines is saturated via salvage pathways in resting cells, proliferating cells depend on the de novo synthesis of pyrimidines to meet the high demand of nucleotides. Here we aim to establish a novel inhibitor PTC299 targeting the rate-limiting enzyme DHODH of the de novo pyrimidine biosynthesis pathway.

Methods: In silico analysis of publically available gene expression data sets was used to analyze the enzymes involved 
in the de novo synthesis of pyrimidines. In vitro, PTC299 was tested in a large panel of MM cell lines. Immunoblotting, flow cytometry and cell based assays (overexpression, knock-out experiments) were used to analyze the underlying mechanisms and specificity of the drug.

Results: In silico gene expression analysis revealed an upregulation of genes associated with pyrimidine biosynthesis in high-risk-MM and linked high expression levels of DHODH to poor patient outcome.

In vitro, inhibition of DHODH induced S-phase cell cycle arrest and apoptosis (cleaved caspase 3 and PARP) accompanied by DNA damage $(\gamma-\mathrm{H} 2 \mathrm{AX})$ and de-regulation of BH3 family members (e.g. upregulation of BIM, BAX and BAK on protein level). Uridine rescue, DHODH knock-out and overexpression experiments confirmed the specificity of PTC299. PTC299 remained effective in models of drug resistance (S63845, Carfilzomib, Pomalidomide).

Conclusions: Our data linked enzymes involved in the de novo pyrimidine biosynthesis to high-risk $\mathrm{MM}$ and evaluated PTC299 as a specific and potent drug targeting DHODH.

\section{POSTER HÄMATOLOGIE - UPDATES ZU 2020}

\section{H20}

Combined targeting of distinct c-Myc and JunB transcriptional programs for multiple myeloma therapy - Update

\section{Judith Lind ${ }^{\star 1}$, Felix Czernilofsky ${ }^{1}$, Sonia Vallet ${ }^{1,2}$, Fengjuan Fan ${ }^{3}$, Latifa Bakiri', Erwin Wagner ${ }^{4,5}$, Martin Sattler6, Martin Pecherstorfer', Klaus Podar ${ }^{1,2}$}

${ }^{1}$ Karl Landsteiner Private University of Health Sciences, Krems, Austria

${ }^{2}$ Department of Internal Medicine II, University Hospital Krems, Krems, Austria

${ }^{3}$ Union Hospital, Tongji Medical College, Huazhong, University of Science and Technology, Wuhan, China ${ }^{4}$ Department of Medical and Chemical Laboratory Diagnostics, Medical University of Vienna, Vienna, Austria ${ }^{5}$ Department of Dermatology, Medical University of Vienna, Vienna, Austria

${ }^{6}$ Dana-Farber Cancer Institute, Department of Medical Oncology, Boston, United States of America

Background: Inhibition of transcription factors (TF) hold great promise as they build convergence points of cell signaling, directing cell proliferation/survival. The TF c-Myc plays a pivotal role in multiple myeloma (MM) and also the TF JunB/AP-1 was attributed a pathophysiologic role in MM. However, approaches to target TFs, such as c-Myc/JunB, are challenged by redundancy phenomena. Former studies demonstrated a key role of c-Myc and JunB, as merging points of the PI3K- and MEK/MAPK-signaling pathways.

Methods: The combined targeting of c-Myc-/JunB-induced transcriptional programs were investigated using knockdown approaches followed by survival, proliferation, flow cytometry, WB, qPCR and luciferase assays.

Results: Inhibiting c-Myc was induced by MZ-1, a novel proteolysis-targeting chimera, specifically targeting BRD4. MZ-1 significantly decreased BRD4/c-Myc levels, inhibited cell growth and survival in cell lines and primary cells. Further, BMSC/IL-6-induced BRD4/c-Myc-upregulation in MM cells was reduced by MZ-1, indicating that targeting BRD4 at least partly overcomes the protective effect of the microenvironment. Notably, MZ-1 did not affect BMSCs/IL-6-induced upregulation of JunB RNA/protein levels. Conversely, knockdown of BMSC/IL-6-triggered JunB upregulation in TetshJunB/ MM.1S cells did not decrease BRD4/c-Myc levels, indicating the co-existence of two distinct, c-Myc-/JunB-mediated proliferative programs. Combination of MZ-1 with JunB knockdown of IL-6-triggered TetshJunB/MM.1S cells reduced c-Myc as well as JunB levels, synergistically inhibited proliferation, and induced cell death. Likewise, combined treatment of MZ-1 and transient siJunB knockdown or MEK inhibitors resulted in similar effects.

Conclusions: Our data demonstrate the existence of two distinct, TF-mediated proliferative programs, supporting a preclinical rationale for combined targeting of c-Myc/JunB.

\section{H21}

Identification of gene targets of mutant C/EBP $\alpha$ reveals a critical role for MSI2 in CEBPA-mutated AML

\section{Elizabeth Heyes ${ }^{\star 1}$, Luisa Schmidt' ${ }^{1}$, Gabriele Manhart ${ }^{1}$, Thomas Eder', Ludovica Proietti', Florian Grebien ${ }^{1}$}

${ }^{1}$ Institute for Medical Biochemistry, University of Veterinary Medicine Vienna, Vienna, Austria

Background: The gene encoding the transcription factor CCAAT/enhancer-binding protein alpha $(\mathrm{C} / \mathrm{EBP} \alpha)$ is mutated in $10-15 \%$ of Acute Myeloid Leukemia (AML) patients. Frameshifts in the CEBPA N-terminus resulting in exclusive expression of a truncated p30 isoform represent the most prevalent type of CEBPA mutations in AML. C/EBP $\alpha$ p30 interacts with the epigenetic machinery, but it is incompletely understood how p30-induced changes cause leukemogenesis. We hypothesized that critical effector genes in CEBPA-mutated AML are dependent on p30-mediated dysregulation of the epigenome.

Methods: We mapped p30-associated regulatory elements by ATAC-seq, C/EBP $\alpha$ - and H3K27ac-ChIP-seq in a myeloid progenitor cell model for $\mathrm{p} 30$-driven AML that enables inducible RNA interference (RNAi)-mediated knockdown of p30. Concomitant p30-dependent changes in gene expression were measured by RNA-seq.

Results: Integrative analysis identified 117 p30-dependent regulatory elements that were associated with 33 strongly downregulated genes upon p30-knockdown. CRISPR/Cas9-mediated mutational disruption of these genes revealed the RNA-binding protein MSI2 as a critical p30-target. MSI2 knockout caused proliferation arrest and terminal myeloid differentiation in p30driven AML cells and delayed leukemia onset in vivo. Furthermore, we validated the human cell line $\mathrm{KO}-52$ as a $\mathrm{C} / \mathrm{EBP} \alpha$ - and MSI2-dependent model for CEBPA-mutated AML.

Conclusions: In summary, this work presents a comprehensive dataset of p30-dependent effects on epigenetic regulation and gene expression and identifies MSI2 as an important effector of the $\mathrm{C} / \mathrm{EBP} \alpha \mathrm{p} 30$ oncoprotein. 


\section{H22 Oral Best Submitted Abstract Hämatologie}

CD19 CAR T-Zelltherapie bei r/r DLBCL - 5 Jahre Erfahrung an der Medizinischen Universität Wien

Philipp Wohlfarth ${ }^{\star 1}$, Nina Worel ${ }^{2}$, Edit Porpaczy', Philipp Staber ${ }^{1}$, Alexander Hauswirth', Wolfgang Sperr ${ }^{1}$, Karoline Gleixner ${ }^{1}$, Marion Heinz ${ }^{1}$, Martina Schlager ${ }^{1}$, Nina Poursadrollah-Wallner ${ }^{1}$, Nicole Hacker ${ }^{1}$, Ingrid Simonitsch-Klupp ${ }^{3}$, Leonhard Müllauer ${ }^{3}$, Marius Mayerhöfer ${ }^{4}$, Martin Heinisch ${ }^{5}$, Anna Grisold ${ }^{6}$, Fritz Leutmezer ${ }^{6}$, Peter Schellongowski ${ }^{1}$, Thomas Staudinger ${ }^{1}$, Winfried Pickl ${ }^{7}$, Ilse Schwarzinger ${ }^{8}$, Eva Jäger ${ }^{8}$, Georg Greiner ${ }^{8}$, Peter Kalhs ${ }^{1}$, Werner Rabitsch ${ }^{1}$, Ulrich Jäger ${ }^{1}$

${ }^{1}$ Universitätsklinik für Innere Medizin I, Medizinische Universität Wien, Wien, Österreich

${ }^{2}$ Universitätsklinik für Blutgruppenserologie und Transfusionsmedizin, Medizinische Universität Wien, Wien, Österreich

${ }^{3}$ Klinisches Institut für Pathologie, Medizinische Universität Wien, Wien, Österreich

${ }^{4}$ Universitätsklinik für Radiologie und Nuklearmedizin, Medizinische Universität Wien, Wien, Österreich ${ }^{5}$ Diagnosezentrum Urania, Wien, Österreich 6Universitätsklinik für Neurologie, Medizinische Universität Wien, Wien, Österreich

${ }^{7}$ Institut für Immunologie, Medizinische Universität Wien, Wien, Österreich

${ }^{8}$ Klinisches Institut für Labormedizin, Medizinische Universität Wien, Wien, Österreich

Grundlagen: Seit 2016 werden an der Medizinischen Universität Wien (MUW) CD19 CAR T-Zellen bei Patienten mit rezidiviertem und/oder refraktärem $(\mathrm{r} / \mathrm{r})$ diffus großzelligem B-Zelllymphom (DLBCL) eingesetzt. Dies ist eine Analyse der bisher innerhalb und außerhalb von Studien gewonnenen Erfahrung an unserem Zentrum.

Methodik: Deskriptive Auswertung hinsichtlich Erkrankungscharakteristika, Therapieverläufe, Ansprechen und Nebenwirkungen aller DLBCL-Patienten, welche zwischen 04/2016 und 01/2021 an der MUW mit CD19 CAR T-Zellen behandelt wurden.

Ergebnisse: Im Beobachtungszeitraum erhielten 32 Patienten (medianes Alter: 61 [Range: 33-79] Jahre; f:m=14:18) mit $\mathrm{r} / \mathrm{r}$ DLBCL eine CD19 CAR T-Zelltherapie. Im Median waren die Patienten mit 4 (1-12) Linien vortherapiert, bei 16 (50\%) Patienten inkludierte dies eine Hochdosischemotherapie + autologe Stammzelltransplantation. Dreißig (94\%) Patienten hatten zum Zeitpunkt der Zellinfusion eine nachweisbare Erkrankung (CS III/IV: $n=12,38 \%$ ), 11 (34\%) Patienten waren auf die letzte Therapielinie refraktär gewesen. Es wurden Tisagenlecleucel $(n=24,75 \%)$, Lisocabtagen maraleucel $(n=6$, $19 \%)$ und Axicabtagen ciloleucel $(n=2,6 \%)$ verabreicht. Sechs (19\%) Patienten entwickelten ein CRS Grad $\geq 2$, welches in drei Fällen einen Intensivstationstransfer notwendig machte. Es wurden keine höhergradigen neurotoxischen Nebenwirkungen (ICANs) beobachtet. Nach einem medianen Follow-up von 9,5 $(0,6-56,5)$ Monaten wurden eine ORR von $65 \%(n=20)$, eine CR-Rate von $35 \%(n=11)$ und eine PR-Rate von $29 \%(n=9)$ als bestes Ansprechen erzielt. Das geschätzte 12-Monatsüberleben nach Kaplan-Meier betrug 62 \% (95\% CI: 43-79\%).

Schlussfolgerungen: Es liegt eine beinahe 5-jährige Erfahrung mit CD19 CAR T-Zellen an der MUW vor, die die Wirksam- keit der Behandlung belegt. Die Überwindung der primären CAR T-Resistenz stellt beim DLBCL die größte Herausforderung für die Zukunft dar.

\section{H23}

Update: Cardiovascular comorbidities in patients with chronic myelomonocytic leukemia

\section{David Raphael Lackner*1, Klaus Geissler ${ }^{1,2}$}

'Sigmund Freud University, Vienna, Austria ${ }^{2}$ Hospital Hietzing, Vienna, Austria

Background: Chronic myelomonocytic leukemia (CMML) is a rare hematopoietic malignancy which is mostly diagnosed in elderly patients who frequently have one or more comorbidities. The clinical significance of cardiovascular comorbidity in patients with CMML is poorly investigated.

Methods: Using data from the Austrian Biodatabase for CMML (ABCMML) we analyzed the prevalence cardiavascular comorbidity and potential correlations with clinical-laboratory features in patients with CMML.

Results: Of 313 data collected, 115/313 (36\%) CMML patients had documented cardiovascular comorbidity. Coronary heart disease $41 / 115(36 \%)$, atrial fibrillation 34/115 (29\%) and hyper-tension 75/115 (64\%) were documented in CMML patients. None of these conditions had a significant impact of survival. Patients with cardiovascular comorbidity were not significantly different from patients without cardiovascular comorbidity regarding WBC counts, hemoglobin levels, platelet counts and the percentages of blood monocytes. Comparing the CMML patient group with and without cardiovascular comorbidities, an increased occurrence of TET2 muta-tions was observed in CMML patients with cardiovascular comorbidities $51 / 85(60 \%)(p=0.036)$.

Conclusions: Our findings show a high prevalence of cardiovascular abnormalities in patients with CMML. A major success of the study was the evidence that TET2 mutations are clustered in CMML patients with cardiovascular disease, confirming an association of the mutation with cardiovascular disease. However, cardiovascular comorbidity does not seem to have a major impact on prognosis in CMML patients.

\section{H24}

\section{Update: The clinical significance of blood coagulation in patients with chronic myelomonocytic leukemia}

\section{Christoph Weinfurtner ${ }^{* 1}$, Klaus Geissler ${ }^{1,2}$ \\ ${ }^{1}$ Sigmund Freud University, Vienna, Austria ${ }^{2}$ Hospital Hietzing, Vienna, Austria}

Background: Chronic myelomonocytic leukemia (CMML) is a rare hematopoietic disease that is usually diagnosed in elderly people. The clinical significance of blood-coagulationdisorders in CMML-patients is poorly investigated.

Methods: Using patients from the Austrian-Biodatabase for CMML (ABCMML) we analyzed the frequency of disturbances of blood-coagulation and potential correlations with clinicallaboratory features in CMML-patients. Data on blood-coagulation were available in 171 patients. Patients with Marcoumar or DOACs were excluded from the analysis. 
Results: Reduced PT-values (Quick) $(<70 \%)$ were found in 45 of 104 (43\%) patients. The median survival of patients with reduced Quick-values was significantly shorter than in patients without coagulation-disorders (19vs. 49 months, $p=0.006$ ). The proportion of patients with hypofibrinogenemia was higher in patients with reduced Quick-values ( $30 \%$ vs $6 \%, p=0.008$ ), but there was no difference in the proportion of patients with documented bleeding ( $19 \%$ vs $16 \%, p=0.819)$. Thrombocytopenia ( $<100 \mathrm{G}$./1) was present in 70 of $152(46 \%)$ patients. These patients also showed significantly shorter survival-times (17 months vs. 49 months, $p<0.000$ ). The proportion of patients with pathological Quick-values ( $53 \%$ vs. $30 \%, p=0.016)$ and the proportion of patients with documented bleeding was significantly higher ( $38 \%$ vs. $9 \%, p=0.012$ ).

Conclusions: Our results show a high prevalence of plasmatic and platelet coagulation-abnormalities associated with laboratory features of advanced CMML. Although CMMLpatients with coagulation-abnormalities have a lower overall-survival, bleeding seemed to be clinically relevant only in CMML-patients with thrombopenia not in patients with low Quick-values. The prognostic value of thrombocytopenia $(<100$ G/l) in CMML-patients was confirmed and Quick-values were identified as prognostic marker.

\section{H25}

\section{Update: Changes in treatment options and} survival in real life during the last three decades in patients with CMML

\section{Julia Reiser ${ }^{\star 1}$, Klaus Geissler ${ }^{1,2}$ \\ ${ }^{1}$ Sigmund Freud University, Vienna, Austria} ${ }^{2}$ Hospital Hietzing, Vienna, Austria

Background: Chronic myelomonocytic leukemia (CMML) is a hematopoietc malignancy in elderly patients. Changes in treatment options and survival over a long period of time are poorly investigated.

Methods: Using data from the "Austrian Biodatabase for CMML" (ABCMML) we analyzed the use of various treatment options and its impact on survival at different time episodes over a period of more than 3 decades.

Results: In 552 patients information regarding OS was available and in 168 of them data regarding treatment. The proportion of patients who were treated with hydroxyurea, cytostatics and azacitidine, respectively was $0 / 0 / 0 \%$ before 1990 , $65 / 19 / 0 \% 1990-1999,50 / 25 / 32 \% 2000-2010$, and $37 / 12 / 53 \%$ after 2010. During these more than three decades median OS increased from 13 to 19,26 and 28 months, respectively (median OS before vs. after 2000 was 16 vs. 27 months, $p=0.047$ ). Collectively, azacitidine treated patients, but not patients treated with the other treatment options, had an improved survival as compared to CMML-patients without azacitidine therapy (19 vs. $25, p=0.042)$. When looking at subgroups the following patient cohorts had a significant survival benefit in association with azacitidine administration: male patients, patients with monocytosis $\geq 10 \mathrm{G} / \mathrm{L}$, patients with hemoglobin $\geq 10 \mathrm{~g} / \mathrm{dl}$ and with mutations in RASopathy genes.

Conclusions: Our results demonstrate an improvement of survival in CMML patients in the new millennium which was associated with the use of azacitidine. Male patients, patients with massive monocytosis, without anemia and patients with hyperactive RAS signaling pathways seem to have the largest benefit from this treatment.

\section{H26}

Aberrant expression of micro-RNA-125a plays a role in the pathogenesis and treatment of chronic myelomonocytic leukemia - Update

\section{Johannes Lorenz Berg*1, Bianca Perfler ${ }^{1}$, Stefan Hatzl', Andreas Reinisch', Gudrun Pregartner², Ingeborg Klymiuk ${ }^{3}$, Sascha Tierling ${ }^{4}$, Klaus Geissler ${ }^{5,6}$, Strobl ${ }^{7}$, Andrea Berghold ${ }^{2}$, Albert Wölfler ${ }^{1}$, Heinz Sill', Armin Zebisch ${ }^{1,8}$}

${ }^{1}$ Division of Hematology, Department of Internal Medicine, Medical University of Graz, Graz, Austria

2Institute of Medical Informatics, Statistics and Documentation, Medical University of Graz, Graz, Austria ${ }^{3}$ Core Facility Molecular Biology, Medical University of Graz, Graz, Austria

${ }^{4}$ Department of Genetics, University of Saarland,

Saarbrücken, Germany

55th Medical Department with Hematology, Oncology and

Palliative Medicine, Hospital Hietzing, Vienna, Austria

${ }^{6}$ Sigmund Freud University, Vienna, Austria

${ }^{7}$ Otto Loewi Research Center, Immunology and

Pathophysiology, Medical University of Graz, Graz, Austria

${ }^{8}$ Otto-Loewi Research Center for Vascular Biology,

Immunology and Inflammation, Division of Pharmacology,

Medical University of Graz, Graz, Austria

Background: Chronic myelomonocytic leukemia (CMML) is an aggressive myeloid neoplasia, which is frequently treated with the hypomethylating agent azacitidine. Here, we studied the role of micro-RNAs (miRs) in CMML development and azacitidine efficacy.

Methods: miR expression profiling by miR-microarray and qPCR, respectively, was performed in purified hematopoietic stem and progenitor cells (HSPCs) of a Kras-G12D-induced CMML mouse model, in 40 human CMML patient specimens, and in nine healthy human HSPC controls. For functional analyses, miR-125a levels were altered using lentiviral overexpression, small hairpin inhibitors (shRNA) as well as CRISPR/Cas9mediated knockout in a series of myeloid/monocytic leukemia cell lines treated with azacitidine. Subsequently, we performed bisulfite sequencing of the miR-125a promoter region, growth curves, BrdU/7-AAD proliferation assays, as well as AnnexinV/7AAD apoptosis assays.

Results: The expression of miR-125a is decreased in HSPCs of human and murine CMML. Functionally, overexpression of miR-125a in myeloid cells with decreased expression of endogenous miR-125a induced apoptosis while inhibiting cellular growth and proliferation. We further show that the decrease of miR-125a is caused by hypermethylation of its promoter region and can be reversed by the treatment with azacitidine. Indeed, analysis of serially obtained primary patient specimens revealed a higher miR-125a expression after azacitidine treatment than before. Importantly, miR-125a silencing by shRNA transfection or CRIPR/Cas9 editing prevented the azacitidine-induced miR125a increase and impaired the cytotoxic effects of this drug.

Conclusions: Expression of miR-125a is decreased in CMML by hypermethylation of its promoter region. Azacitidine treatment increases miR-125a expression, which contributes to the anti-leukemic efficacy of this drug. 


\section{POSTER ONKOLOGIE}

\section{1}

\section{Seasonal impact on subcutaneous port infections in oncological patients}

\section{Sebastian Ecker*1, David KiesI ${ }^{\star 1}$, David Fuchs² \\ ${ }^{1}$ Department of Hematology and Internal Oncology, Kepler University Hospital, Linz, Austria \\ ${ }^{2}$ Department of Palliative Medicine, Ordensklinikum Linz, Barmherzige Schwestern, Linz, Austria}

Background: Catheter-related bloodstream infection (CRBSI) are among the most frequent complications in neutropenic cancer patients and associated with a high mortality rate. Clinical observations and current literature suggests a relationship between season and detected infection rates.

Methods: We enrolled 1.773 oncological and hematological patients (1.172 female, 601 male) in a retrospective cohort study between 2010-2017 at the University Clinic of Hematology and Internal Oncology at the Kepler University Hospital in Linz, Austria.

Results: 795 of the enrolled patients underwent planned or unplanned Port explantation (Port-Ex). There were 176 Port-Ex in the warm season (June, July, August) and 619 Port-Ex in the cold season group (September-May). From the total number of Port-Ex we observed 98 CRBSI, representing a rate of $12.32 \%$. A statistically significant rise of CRBSI could be detected during summer (18.75\% vs. $10.50 \%)$ representing an increase of $78 \%$. Additionally, a logistic regression analysis determined 'season' to be an important predictor for Port infection with a hazard ratio of 1.785 (CI: $95 \% 1.105$ to $2.885, P$ value $=0.0179$ ) in the warm season.

Conclusions: We demonstrated a highly significant increase of CRBSI in the warm season compared to the cold season. Among the many complications which can occur during the lifetime of a central venous catheter, infection is among the most severe complications, especially in this vulnerable group of patients. The findings of this study should initiate discussion about further assessment of Port infections regarding causes, management and possible prophylactic regimes.

\section{2}

miR-22-3p is a potential novel therapeutic strategy in treating colorectal cancer metastasis

\section{Dominik Barth*1, Felix Prinz ${ }^{1}$, Christiane Klec ${ }^{1}$, George} A. Calin ${ }^{2}$, Martin Pichler ${ }^{1,3}$

1Division of Oncology, Department of Internal Medicine, Medical University of Graz, Graz, Austria

${ }^{2}$ Department of Translational Molecular Pathology, The University of Texas MD Anderson Cancer Center, Houston, Texas, USA

${ }^{3}$ Department of Experimental Therapeutics, The University of Texas MD Anderson Cancer Center, Houston, Texas, USA

Background: Previous studies indicate a tumor suppressive role of miR-22-3p in colorectal cancer (CRC). The related molecular mechanisms and the potential as a therapeutic RNA have not been tested yet.
Methods: In a series of in vitro and in vivo models and molecular analyses, we characterized the fundamental biological roles of miR-22-3p in CRC cell lines. We further explored the therapeutic potential of miR-22-3p in a mouse model of colorectal cancer metastases.

Results: miR-22-3p expression was downregulated in CRC tumor tissue as compared to healthy colorectal mucosa. Cell culture data confirmed a growth inhibitory effect of miR-22-3p in three different cell lines (HCT116, DLD-1 and SW480), that is explained by induction of apoptosis in independent colorectal cancer cells. Mechanistically, miR-22-3p reduced the expression and directly interacts with the anti-apoptotic long noncoding RNA FLANC. In vivo pharmacological targeting of colorectal cancer metastases by administration of nano-liposomal encapsulated miR-22-3p mimics, induced significant decrease in metastases, reduced proliferation and micro vessel density and induction of apoptosis. Importantly, these effects were observed without evident tissue toxicity or pro inflammatory effects in the mouse model.

Conclusions: Based on our findings, we propose miR-22-3p as a potential novel therapeutic strategy in targeting colorectal cancer metastases.

\section{3}

Impact of skeletal muscle dynamics on clinical outcome in advanced non-small cell lung cancer (NSCLC) patients undergoing immune-checkpoint blockade: A retrospective study

\section{Benedikt Jörg ${ }^{\star 1}$, Florian Huemer ${ }^{1}$, Richard Greil' Gabriel Rinnerthaler*1}

1Department of Internal Medicine III, Salzburg Cancer Research Institute, Paracelsus Medical Private University, Salzburg, Austria

Background: In this retrospective analysis, we evaluated the impact of skeletal muscle dynamics on clinical outcome in advanced NSCLC patients undergoing palliative therapy with immune-checkpoint inhibitors (ICI).

Methods: Medical records of advanced NSCLC patients with ICI either as monotherapy or in combination with chemotherapy (CTx) between May 2015 and June 2020 at our tertiary cancer center were reviewed. Skeletal muscle index (SMI) was obtained from cross-sectional images of routinely performed computed tomography (CT) scans and compared between initiation of ICI therapy and after 2-3 months.

Results: Among 74 included advanced NSCLC patients, $47(64 \%)$ presented with sarcopenia at ICI therapy initiation. Presence of baseline sarcopenia did not impact on median OS in the entire NSCLC cohort (12.4 versus 15 months, $p=0.58$ ), the combined CTx + ICI cohort (median OS 10.4 versus 11.5 months, $p=0.37$ ) or the ICI monotherapy cohort (median OS 19.5 versus 16.0 months, $p=0.77)$. However, baseline sarcopenia was associated with a decreased TTF (median TTF 3.9 versus 6.0 months, $p=0.0018)$. An increase of SMI during ICI therapy predicted superior OS in the entire cohort $(28.0$ versus 11.0 months, $p<0.001$ ), combined CTx + ICI cohort ( 18.6 versus 10.8 months, $p=0.033$ ) and ICI monotherapy cohort (33.0 versus 13.5 months, $p=0.027)$, respectively. An SMI increase was also associated with longer TTF ( 7.8 versus 5.5 months, $p<0.001$ ).

Conclusions: Due to the favorable clinical outcome associated with skeletal muscle increase during ICI therapy, serial assessments of SMI may help to guide therapeutic decision making in NSCLC patients with equivocal radiologic response. 
004

Pitfall in immunotherapy: sarcoid like reaction mimicking disease progression in advanced melanoma treated with nivolumab

\section{Beatrix Mutschlechner*1, Susanne Dertinger ${ }^{1}$, Felix Offner ${ }^{1}$, Veronika Petz ${ }^{1}$, Alexander Becherer ${ }^{1}$, Klaus Gasser ${ }^{1}$, Bernd Hartmann', Thomas Winder ${ }^{1}$}

${ }^{1}$ Academic Teaching Hospital Feldkirch, Feldkirch, Austria ${ }^{2}$ Private University in the Principality of Liechtenstein, Triesen, Principality of Liechtenstein

${ }^{3}$ University of Zurich, Zurich, Switzerland

Immunotherapy is the first-line treatment in many solid organ carcinomas. The activation of the immune system leads to specific immune related adverse events (irAE), which also include the rarely occurring sarcoid like reaction (SLR). In imaging studies SLR can mimic carcinomatous lesions and thus simulate disease progression. Even though most patients suffering from SLR are asymptomatic and do not need anti-sarcoidosis therapy, it is crucial to not misdiagnose irAE as disease progression and unnecessarily discontinue vital immunotherapy. The first occurrence of SLR can usually be noticed soon after the start of immunotherapy. However, as previously described in literature, our case report shows that SLR can also occur months after the start of immunotherapy. This is why SLR should be considered at any point during the disease course, if progression of the disease is suspected. In addition, the diagnosis of SLR is complicated by the fact that it can only be confirmed histologically, since neither the patient's symptoms, nor serum markers, nor clear imaging features are conclusive for SLR. In this case report we want to highlight the clinical relevance of recognizing SLR in a patient with advanced melanoma treated with nivolumab and emphasize the importance of re-biopsy in case of suspected disease progression, especially in less plausible cases. In the end, identifying SLR remains difficult and requires high knowledge and interdisciplinary cooperation.

\section{5}

Poor outcome in patients with active malignancy and severe or critical COVID-19. A multicentre registry

\section{Simon Udovica ${ }^{\star 1}$, Nino Müser ${ }^{1}$, Agnes Pechlaner ${ }^{2}$, Andreas Reichinger ${ }^{3}$, Florian Vafai-Tabrizi ${ }^{4}$, Ewald Wöll', Holger Rumpold ${ }^{5,6}$, Andreas Petzer ${ }^{3}$, Wolfgang Hilbe' ${ }^{1}$, Ercan Müldür ${ }^{1}$}

${ }^{1}$ Department of Medicine I, Wilhelminen Cancer Research Institute, Klinik Ottakring, Vienna, Austria

${ }^{2}$ Department of Internal Medicine, St. Vinzenz Hospital, Zams, Austria

${ }^{3}$ Department of Hematology and Medical Oncology, Ordensklinikum Linz, Linz, Austria

${ }^{4}$ Department of Internal and Respiratory Medicine, Wilhelminenspital, Vienna, Austria

${ }^{5}$ Gastrointestinal Cancer Center, Ordensklinikum Linz, Linz, Austria

${ }^{6}$ Medical Faculty, Johannes Kepler University, Linz, Austria

Background: Patients with cancer are considered to be at higher risk for COVID-19 related death compared to patients without cancer. We analysed data from patients with active malignancy who were hospitalised with COVID-19 at three oncologic centres in Austria.

Methods: 165 patients hospitalised with COVID-19 between March and December 2020 were included in the analysis. 75 patients were diagnosed with active malignancy, 44 of those suffered from severe or critical COVID-19. As a control group, we analysed 90 patients without history of cancer and treated for COVID-19 at the centre in Vienna. Among those, 74 patients had severe or critical COVID-19. Prediction of in-hospital mortality was performed using the 4c Mortality Score. Primary endpoint was COVID-19 related in-hospital mortality. Fisher's exact test was used for univariate analysis.

Results: In patients with severe or critical COVID-19, mortality was significantly higher in cancer patients than in controls $(47.50 \%$ vs. $24.32 \%, p=0.02)$. Moreover, observed mortality was higher than predicted in cancer patients $(47.50 \%$ vs. $30.23 \%$ ), but similar in the control cohort ( $24.32 \%$ vs. $22.70 \%)$. Frequency of critical COVID-19 (31.82\% vs. $33.78 \%)$, ICU rate ( $13.64 \%$ vs. $13.51 \%$ ), age (median: $73 a$ vs. $72 a$ ) and number of comorbidities (mean: 1.64 vs. 1.72) were comparable between the two groups. No cancer patient with mild or moderate COVID-19 died.

Conclusions: Our data indicate that active malignancy is an independent risk factor for COVID-19 associated mortality in patients with severe or critical COVID-19. Encouragingly, however, we also observed 31 cancer patients with only mild to moderate COVID-19.

\section{Gewinner Jubiläumspreis}

Benefit of second-line therapy for advanced esophageal squamous cell carcinoma: A tri-centre propensity score analysis

Moritz Müller ${ }^{\star 1}$, Florian Posch', Dominik Kiem², Dominik Barth', Lena Horvath ${ }^{3}$, Christopher Lobenwein ${ }^{3}$, Michael Stotz', Renate Schaberl-Moser', Martin Pichler', Richard Greil', Philipp Jost', Andreas Seeber ${ }^{3}$, Arno Amann ${ }^{3}$, Armin Gerger ${ }^{1}$, Konstatin Schlick ${ }^{2}$, Jakob M. Riedl'

1'Division of Oncology, Department of Internal Medicine, Medical University of Graz, Graz, Austria

${ }^{2}$ IIIrd Medical Department of Hematology, Medical Oncology, Hemostaseology, Rheumatology and Infectious Disease, Salzburg Cancer Research Institute, Paracelsus Private Medical University, Salzburg, Austria

${ }^{3}$ Department of Internal Medicine V: Hematology and Oncology, Medical University of Innsbruck, Innsbruck, Austria

Background: The level of evidence for established treatment regimens in the 2nd line therapy (2LTX) of advanced esophageal squamous cell carcinoma (aESCC) is limited. This is the first study that reports efficacy data comparing 2LTX versus active symptom control (ASC) in aESCC.

Methods: We conducted a tri-centre retrospective cohort study $(n=166)$, including all consecutively treated patients with aESCC who had experienced disease progression on palliative 1 st line therapy (1LTX). A propensity score model using inverseprobability-of-treatment-weighting (IPTW) was implemented for comparative efficacy analysis of overall survival (OS) in patients with 2LTX+ASC versus ASC. 
Results: After progression on 1LTX, 74 (45\%) patients were treated with ASC while $92(55 \%)$ received 2LTX+ASC. The most frequent 2LTX regimens were docetaxel (36\%) and paclitaxel (18\%). In unadjusted analysis, 2LTX+ASC was associated with significantly longer OS compared to ASC only. However, patients in the 2LTX+ASC had a significantly higher prevalence of favourable prognostic factors such as better ECOG performance status, longer duration of 1LTX and lower baseline CRP levels. These imbalances could be fully removed by re-weighting the data with the IPTW. In the IPTW-adjusted analysis the favourable association between 2nd-line therapy and longer overall survival weakened but prevailed. The median OS was 6.1 months in the 2LTX group and 3.2 months in the ASC group, respectively (IPTW-adjusted Hazard ratio $=0.40$, 95 \%CI: $0.24-$ $0.69, p=0.001$ ). Importantly, the benefit of 2LTX was consistent across several clinical subgroups, including patients with ECOG performance status $\geq 1$ and age $\geq 65$.

Conclusions: This real-world study supports the concept that 2LTX prolongs survival in patients with aESCC.

\section{7}

Efficacy of oncological treatment in metastatic colorectal cancer is independent of comorbidities and age

Dora Beke-Niedersüß1', Manuel Orlinger², David Falch', Josef Thaler ${ }^{3}$, Wolfgang Hilbe ${ }^{1}$, Andreas Petzer ${ }^{2}$, Holger Rumpold ${ }^{\star 4,5}$

'Department of Internal Medicine I, Wilhelminenspital, Vienna, Austria

2Department of Hematology and Medical Oncology, Ordensklinikum Linz, Linz, Austria

${ }^{3}$ Department of Internal Medicine IV, Hospital Wels-

Grieskirchen, Wels, Austria

${ }^{4}$ Gastrointestinal Cancer Center, Ordensklinikum Linz, Linz,

Austria

${ }^{5}$ Medical Faculty, Johannes Kepler University Linz, Linz,

Austria

Background: Colorectal Cancer (CRC) belongs to the most common malignancies. Treatment decisions depend on comorbidities age. We aimed to investigate the impact of comorbidities and age on efficacy of oncological treatments.

Methods: Patients with metastatic CRC (mCRC) diagnosed between Jan 1st 2007 and 31st Dec 2019 were included. Charlson Comorbidity Index (CCI) age-adjusted CCI (aaCCI) were determined by chart review; cancer as comorbidity was omitted for CCI/aaCCI calculation.

Results: In total, 1015 patients were included; CCI/aaCCI was available from 566 patients. Median follow up was 76 months (CI $41.46 ; 107.13)$. aaCCI $>3$ was of highest prognostic value compared to other aaCCI-levels, CCI or age $(p<0.001$ for all). Comorbid or older patients were significantly less often treated (aaCCI $>3: 37.6 \%$ vs $12.4 \%$; age $>70$ years: 35.7 vs $11.2 \%$; both $p<0.001$ ). Treatment (best supportive care [BSC]; systemic treatment only [STO]; resection of metastases [ROM]) significantly interacted with overall survival (OS; 6 vs 19.8 vs 54.3 months; $\mathrm{p}$ for interaction $<0.001)$. Neither treatment approach, however, interacted significantly with either aaCCI $>3$ (BSC $p=0.46$; STO $p=0.23$; ROM $p=0.52$ ) or age (BSC $p=0.16$; STO $p=0.18 ; \operatorname{ROM} p=0.90$ ).

Conclusions: Patients with comorbidities or higher age are significantly less often treated. Efficacy of oncological treatment, however, is independent of comorbidities or age. Carful patient selection is mandatory, but balancing risk and benefit should take potential undertreatment of comorbid and older patients into account.

\section{8}

Internal validation of residual cancer burden as a prognostic factor and its correlation with chemotherapy doses in breast cancer patients undergoing neoadjuvant therapy

\section{Christoph Suppan*1, Nina Mischitz ${ }^{1}$, Daniel Steiner ${ }^{1}$, Florian Posch ${ }^{1}$, Eva Valentina Klocker ${ }^{1}$, Hannah Deborah Müller', Hubert Hauser ${ }^{2}$, Robert Hammer², Ute Bargfrieder ${ }^{3}$, Sigurd Lax ${ }^{3}$, Nadia Dandachi' ${ }^{1}$, Marija Balic ${ }^{1}$}

${ }^{1}$ Division of Oncology, Department of Internal Medicine, Medical University Graz, LKH Graz, Graz, Austria ${ }^{2}$ Department of Surgery, LKH Graz II, Standort West, Graz, Austria

${ }^{3}$ Institute of Pathology, LKH Graz II, Standort West, Graz, Austria

Background: Residual Cancer Burden (RCB) has been confirmed as an independent prognostic marker for survival in breast cancer patients undergoing neoadjuvant therapy. However, the association of chemotherapy dose reduction and higher RCB scores is unknown.

Methods: Between 2011 and 2020 the RCB scores of 367 female breast cancer patients were prospectively assessed. Clinico-pathological and follow up data were obtained retrospectively. Recurrence-free survival (RFS), overall survival (OS) and distant-disease free survival (DDFS) were calculated. To enable an analysis of neoadjuvant dose density, we computed standardized cumulative doses of anthracyclines and taxanes (A/T).

Results: Higher RCB scores were associated with worse RFS, worse DDFS, and worse OS $(p<0.0001)$. Clinical outcome of patients with RCB class 1 did not differ substantially at 5 years compared to RCB 0 . We also observed proportionality of hazards according to the RCB score, with a peak for risk of relapse between 1-2 years after surgery. 180 patients $(49.1 \%)$ underwent dose reduction of neoadjuvant A/T chemotherapy. An interaction between dose reduction impacting higher RCB values has been observed (interaction $p$-value $=0.042$ ).

Conclusions: Our results confirm RCB as a prognostic marker for RFS and OS independent of molecular subtype. Importantly, lower doses of cumulative neoadjuvant A/T were associated with higher RCB scores in patients who required a dose reduction. 


\section{O09 Gewinner Jubiläumspreis}

Incidence, management and clinical outcomes of oncological patients with SARS-2 CoV-2 infection during the second COVID-19 pandemic wave in South Tyrol

\section{Manfred Mitterer ${ }^{\star 1}$, Dominic Fong1, Katja San Nicolo', Maximilian Mair ${ }^{2}$, Thomas Buratti ${ }^{1}$, Monika Alber ${ }^{1}$ \\ 'Oncology Outpatient Department, „Franz Tappeiner“ Hospital Meran, Meran, Italy \\ 2Division of Oncology, Department of Medicine I, Medical University of Vienna, Vienna, Austria}

Background: The impact of the "second Covid-19 pandemic wave" in haemato-oncology patients in a real-world setting is largely unknown.

Methods: Between Oct 19, 2020 and Jan 29, 2021, 5011 visits were performed and 1535 systemic therapies were administered at the haemato-oncology Day Hospital Meran (South Tyrol, Italy). Additional preventive SARS-CoV-2 measures included a questionnaire for COVID-19-specific symptoms as well as a SARS-CoV-2 RT-PCR two days prior to intravenous therapy. In addition, evaluation of anti-SARS-CoV-2 antibodies and lymphocyte subsets was performed.

Results: Out of 2297 RT-PCR tests performed, a total of 101 ( $4.4 \%)$ patients tested positive for SARS-CoV-2. No SARS-CoV-2 transmission occurred within the hospital. Within the first three weeks, the incidence in the general population was similar to that of our patient cohort $(18.8 \%$ vs $18.6 \%)$, while thereafter it decreased by $>50 \%$ ( $11.2 \%$ vs $4.3 \%)$. Overall inpatient hospital admission was $18 \%$ (general COVID-ward $16 \%$, ICU $2 \%$ ) whereas $5 \%(5 / 101)$ SARS-CoV-2 positive patients died. The median duration of SARS-CoV-2 positivity was 16 days (range: 7-64). Anti-nucleocapsid and anti-spike IgG-antibodies were absent in $25 \%$ and $35 \%$ of patients, respectively. Peripheral CD4+, CD20+ and CD8+ lymphocytes were decreased in $26 \%$, $34 \%$ and $45 \%$ of SARS-CoV-2 positive patients.

Conclusions: Although the incidence of SARS-CoV-2 infections was high in the second COVID-19 pandemic wave, continuing cancer care seems to be safe due to maintenance of specific infection control measures. The low seroprevalence and impaired adaptive immunity in infected cancer patients warrants further investigation.

\section{Gewinner Jubiläumspreis}

\section{Clinical relevance of HER2 testing in breast cancer patients treated at the Division of Oncology in Graz}

\section{Daniel Steiner ${ }^{* 1}$, Eva Valentina Klocker ${ }^{1}$, Christoph Suppan' ${ }^{1}$, Florian Posch'1, Hannah Deborah Müller' ${ }^{1}$, Marija Balic ${ }^{1}$, Nadia Dandachi'}

'Division of Oncology, Department of Internal Medicine, Medical University of Graz, Graz, Austria

Background: Breast cancer poses a major public health issue. HER2 expressing breast cancer can be targeted with established therapeutic options, therefore definition and assessment of HER2 positivity is of clinical relevance.

Methods: We conducted a single-center, retrospective data analysis at the Division of Oncology, Graz. Patients were eligible having invasive breast cancer with a HER2 IHC score of 2+ or 3+ between 2006 and 2016. We assessed the distribution of molecular subtypes in our patients. Primary end point was clinical outcome in HER2 positive patients stratified by receipt of HER2 targeted therapy. Secondary end point was number of patients affected by the change in ASCO/CAP guidelines regarding HER2 positivity and resulting influence on therapeutic decisions and outcomes.

Results: Of 611 patients included, 353 had a score of $2+$ and 258 of $3+$, resulting in 343 HER2 positive, 264 HER2 negative, and 4 unknown cases. OS at 10 years for HER2 positive patients in (neo)adjuvant setting was significantly better when patients received HER2 targeted therapy $(81.38 \%$ vs. $63.48 \% ; p<0.005)$. Of 353 patients with a score of $2+, 12$ had a HER2/CEP17 ratio between 2.0 and 2.2. Due to the low case number, the influence on therapeutic decisions and outcomes could not be determined.

Conclusions: Breast cancer patients with a HER2 IHC score of $2+$ and $3+$ are a diverse population. In our study, HER2 targeted therapy was a crucial component in treating HER2 positive patients. The clinical importance of the change of the ASCO/CAP guidelines could not be determined.

\section{KLINISCHE STUDIEN}

\section{S01}

CaboPoint: a phase II study of second-line cabozantinib in patients with metastatic renal cell carcinoma (RCC)

\section{Manuela Schmidinger*1, Laurence Albiges ${ }^{2}$, Naila Taguieva-Pioger ${ }^{3}$, David Perol $^{4}$, Viktor Grünwald ${ }^{5}$}

${ }^{1}$ Division of Oncology, Department of Medicine I, Medical University of Vienna, Vienna, Austria

${ }^{2}$ Medical Oncology, Gustave Roussy, Université ParisSaclay, Villejuif, France

${ }^{3}$ Ipsen, Boulogne-Billancourt, France

${ }^{4}$ Department of Clinical Research, Léon Bérard Cancer Centre, Lyon, France

${ }^{5}$ University Hospital Essen, West-German Cancer Center, Clinic for Internal Medicine (Tumor Research) and Clinic for Urology, Essen, Germany

Background: Cabozantinib is an approved therapy for advanced RCC (USA) and in treatment-naïve patients with intermediate/poor risk, as well as following VEGF-targeted therapy (Europe).

Trial design: CaboPoint (NCT03945773) is a phase II, open-label study of cabozantinib in adults with unresectable, locally advanced or metastatic clear-cell RCC, whose disease progressed after checkpoint inhibitor therapy with ipilimumab and nivolumab alone (cohort A) or in combination with VEGFtargeted therapy (cohort B). The primary endpoint is objective response rate. Secondary endpoints include time to response, duration of response, disease control rate, progression-free survival and overall survival. A target of 250 patients at 50 European sites will receive cabozantinib ( $60 \mathrm{mg}$ once daily; self-administered at home) for $\leq 18$ months after the last patient receives their first dose. Patients may continue cabozantinib after disease progression if there is clinical benefit. During follow-up, patients who discontinue early will be contacted every 12 weeks 
to assess survival and subsequent therapy. Interim analysis when $60 \%$ of a cohort reach 12 months of follow-up.

CaboPoint has been adjusted to allow the trial to continue during the COVID-19 outbreak, protecting participants in compliance with the study protocol.

Alternative arrangements include (if participant cannot attend the study site): study drug dispensation to the participant's home; safety assessments remotely at a local health care provider; assessments at a local radiology facility.

Enrolment is permitted if the patient can be managed in compliance with the protocol and alternative arrangements.(1)

Funded by Ipsen.

Sources:

(1) https://ec.europa.eu/health/sites/health/files/files/ eudralex/vol-10/guidanceclinicaltrials_covid19_en.pdf

\section{SO2}

GELTAMO18-HL:

BRESELIBET - BREntuximab Vedotin in SEcond LIne Therapy BEfore Transplant

\section{Richard Greil $^{\star *}$, Thomas Melchardt ${ }^{1}$}

1'Department of Internal Medicine III with Haematology, Medical Oncology, Haemostaseology, Infectiology and Rheumatology, Oncologic Center, Paracelsus Medical University Salzburg, Salzburg, Austria

Study Title: A Randomized Phase IIb Study, Evaluating Efficacy of Salvage Therapy with Brentuximab Vedotin-ESHAP vs ESHAP in Patients with Relapsed/Refractory Classical Hodgkin's Lymphoma, Followed by Brentuximab Vedotin Consolidation (instead of Autologous Hematopoietic Stem Cell Transplantation) in Those who Attained a Metabolic Complete Remission after Salvage Therapy

Design: The first part of the study randomizes 3 cycles of ESHAP as a standard of care therapy for those patients with primary refractory $\mathrm{cHL}$ and those patients relapsing after first-line therapy versus 3 cycles ESHAP-BV with BV at a dose of $1.8 \mathrm{mg} /$ $\mathrm{kg}$ IV. The rationale behind this first part is the lack of prospective randomized comparisons between conventional salvage chemotherapy protocols and its BV-containing counterpart in terms of rate of metabolic CRs and ORR.

In the second part of the trial, those patients that achieve a mCR will be consolidated with either 13 or 16 cycles of BV (depending on the prior treatment yes/no with BV together with the salvage second-line strategy) at the usual doses and time intervals ( $1.8 \mathrm{mg} / \mathrm{kg}$ iv in $30 \mathrm{~min}$ every 21 days). The objective of this second part is to try to avoid auto-HCT as well as early, mid and long-term toxicities associated to the procedure in a population of patients that have a better prognosis and substitute it by an anti-CD30 monoclonal-antibody drug conjugate that has demonstrated a beneficial effect as a consolidation strategy in the AETHERA Trial.

\section{SO3}

\section{Pola-R-ICE:}

An open-label, prospective-Phase-III clinical study to compare polatuzumab vedotin+rituximab+if osfamide+carboplatin+etoposide (Pola-R-ICE) with rituximab+ifosfamide+carboplatin+etoposide (R-ICE) alone as salvage-therapy in patients with primary refractory or relapsed diffuse-large-Bcell-lymphoma (DLBCL)

\section{Richard Greil¹}

1Department of Internal Medicine III with Haematology, Medical Oncology, Haemostaseology, Infectiology and Rheumatology, Oncologic Center, Paracelsus Medical University Salzburg, Salzburg, Austria

Design: International, multicenter, open-label, two-arm, randomized, prospective, phase III study with Polatuzumab vedotin plus rituximab, ifosfamide, carboplatin and etoposide (Pola-R-ICE) versus R-ICE alone in second line treatment of diffuse large B-cell lymphoma (DLBCL).

Population: Male and female subjects 18 years (16 years in the UK) or older suffering from first relapse or primary refractory disease of DLBCL.

Primary objective: The primary objective of this study is to investigate the following question in patients with relapsed or primary refractory DLBCL: Does salvage therapy with Pola-RICE improve event-free survival (EFS) compared to R-ICE alone?

Primary endpoint: The primary endpoint is EFS of patients with DLBCL at first progression or relapse. EFS is defined as the time between the day of randomization and the occurrence of any of the following events:

- Failure to achieve sufficient response in PET-CT (Deauville score 3 or less) at end of study treatment (metabolic CR)

- Disease progression (PD)

- Start of additional unplanned anti-tumor treatment (radiation therapy allowed)

- Relapse after achieving CR

- Death due to any cause

Patients who have not experienced any of these events by the time of analysis will be censored at the most recent date of disease assessment. 
Keeping haemato-oncology health care workers Covid-19 safe: success and failure

Michele Atzl ${ }^{\star 1}$, Andreas Leiherer,3, Axel Mündlein², Beatrix Mutschlechner ${ }^{1}$, Magdalen Benda ${ }^{1}$, Lukas Sprenger ${ }^{1}$, Andreas Volgger ${ }^{1}$, Luciano Severgnini ${ }^{1}$, Johannes Jäger ${ }^{1}$, Alois Lang ${ }^{4}$, Thomas Winder ${ }^{1}$

'Division Haematology, Oncology, Gastroenterology and Infectious Diseases, Department of Internal Medicine, Academic Teaching Hospital, Feldkirch, Austria

${ }^{2}$ Vorarlberg Institute for Vascular Investigation and Treatment (VIVIT), Feldkirch, Austria

${ }^{3}$ Private University of the Principality of Liechtenstein, Triesen, Liechtenstein

${ }^{4}$ Department of Internal Medicine, Lindenberg Hospital, Lindenberg, Germany

Background: During the SARS-CoV-2 pandemic, health care workers (HCW) may be at higher risk of contracting the disease. We introduced stringent measures to maintain a Covid19free environment. Here we report the seroprevalence amongst HCW in our cancer unit.

Methods: $54 / 56$ of our staff voluntarily enrolled to a larger prospective Covid-19 seroprevalence survey in HCW in Vorarlberg. Three monthly serum specimens were tested for the presence of anti-SARS-CoV-2 IgG antibodies specific for virus NP and RBD. We collected clinical information and household demographics. Baseline was 3 months after the first wave of the epidemic that occurred in March 2020, the second round before the second wave of the epidemic in October, and the latest round immediately before beginning of the Covid-19 vaccination program in January 2021.

Results: Our first PCR positive case by means of nasopharyngeal swab amongst our team occurred on 26.10.2020. By January 2021, altogether 12/54 HCW participants in our unit had tested antibody positive. $9 / 12$ team members who contracted SARS-CoV2 had persons $<25$ yrs in the household, whereas only $3 / 25$ HCW living in households without persons $<25$ yrs, contracted Covid-19 disease.

Conclusions: The first round of internal and community measures was successful, with no cases after the first wave of the epidemic. The second wave, which preserved the same measures internally, but was not accompanied by the same harsh community restrictions, experienced a higher number of HCW SARS-CoV-2-cases in our unit. Community spread probably contributed to cases amongst our HCW.

Publisher's Note Springer Nature remains neutral with regard to jurisdictional claims in published maps and institutional affiliations.

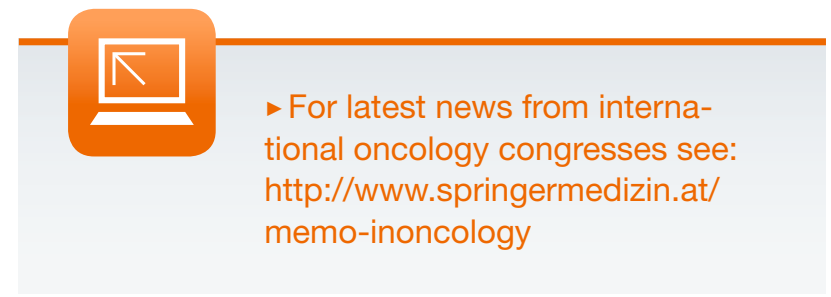

